\title{
Plant-Based Food By-Products: Prospects for Valorisation in Functional Bread Development
}

\author{
Isaac Amoah 1,2,3,*, Noamane Taarji 4,5, Paa-Nii T. Johnson ${ }^{6}{ }^{\circledR}$, Jonathan Barrett ${ }^{1}$, \\ Carolyn Cairncross ${ }^{1}$ and Elaine Rush ${ }^{1,2, *(D)}$ \\ 1 Faculty of Health and Environmental Sciences, Auckland University of Technology, \\ Auckland 1010, New Zealand; tbx4675@autuni.ac.nz (J.B.); carolyn.cairncross@aut.ac.nz (C.C.) \\ 2 Riddet Institute, Centre of Research Excellence, Palmerston North 4442, New Zealand \\ 3 Department of Biochemistry and Biotechnology, Kwame Nkrumah University of Science and Technology, \\ Kumasi 0023351, Ghana \\ 4 Food and Medicinal Resource Engineering Open Innovation Laboratory, National Institute of Advanced \\ Industrial Science and Technology (AIST), Tsukuba City, Ibaraki 305-8565, Japan; taarji.noamane@aist.go.jp \\ 5 Faculty of Sciences Semlalia, Cadi Ayyad University, P.O. Box 2390, 40001 Marrakech, Morocco \\ 6 Department of Agroprocessing Technology and Food Biosciences, CSIR-College of Science and Technology, \\ Accra Box M20, Ghana; paanii.johnson@gmail.com \\ * Correspondence: isaacamoah458@gmail.com or isaac.amoah@aut.ac.nz (I.A.); elaine.rush@aut.ac.nz (E.R.); \\ Tel.: +64-2108659403 or +23-3249183185 (I.A.); +64-21-624-077 (E.R.)
}

Received: 19 August 2020; Accepted: 15 September 2020; Published: 21 September 2020

check for updates

\begin{abstract}
The industrial and small-scale processing of plant-based food materials is associated with by-products that may have a negative impact on the environment but could add value to bread-based products. The bioactivity of plant-based food by-products, their impact on the properties of functional bread, and their bioavailability/bioaccessibility leading to potential health effects when consumed was reviewed. Plant-based food by-products which may be added to bread include rice bran, wheat bran, corn bran, grape pomace/seed extract, tomato seed/skin, and artichoke stems/leaves. These by-products contain high concentrations of bioactive compounds, including phenolics, bioactive peptides, and arabinoxylan. Pre-treatment procedures, including fermentation and thermal processing, impact the properties of plant-based by-products. In most cases, bread formulated with flour from plant-based by-products demonstrated increased fibre and bioactive compound contents. In terms of the sensory and nutritional acceptability of bread, formulations with an average of $5 \%$ flour from plant-based by-products produced bread with acceptable sensory properties. Bread enriched with plant-based by-products demonstrated enhanced bioavailability and bioaccessibility and favourable bioactive properties in human blood, although long-term studies are warranted. There is a need to investigate the bioactive properties of other underutilised plant-based by-products and their potential application in bread as a sustainable approach towards improving food and nutrition security.
\end{abstract}

Keywords: plant-based food by-product; bread; bioactivity; bread qualities; sensory properties; bioavailability; bioaccessibility

\section{Introduction}

Industrial and small-scale processing of plant-based food materials generate by-products including wheat bran [1], grape pomace [2,3], pineapple pomace [4,5], broccoli by-products (stalk and leaves) [6], and oil seed cakes from flaxseed [7] and naked pumpkin seed/sunflower seed/yellow linseed/walnut [8]. These plant-based food by-products have low economic value as they are either mostly fed to livestock [9] or discarded in landfill sites [10]. Yet, the large quantum of plant-based food by-products generated by the food industry warrants the need for exploitation of their potential use in new food 
product development including bread. For example, the wheat and rice milling industry globally generates 90 [1] and 29.3 [11] million tonnes, respectively, of wheat and rice bran annually. Pineapple processing generates pomace which constitutes $30 \%$ of the pineapple fruit pulp (fresh weight) [5] and the grape juice industry produces pomace which constitutes up to $20 \%$ of the total weight of grapes [12].

Disposal of plant-based by-products from the food industry is an arduous task creating a burden on the environment as it results in the generation of greenhouse gases including carbon dioxide and methane and additionally diminishes potential economic gains by the food industry [10]. The search for innovative technologies geared towards valorising plant-based food by-products for food product development is currently of research interest. Consequently, the application of pre-treatment processes to these plant-based food by-products, including fermentation [13-15], freeze-drying [16], oven-drying [17], and other thermal treatments, have been explored to enhance the bioactive and aromatic profiles of plant-based food by-products, improve their techno-functional properties, and increase their potential to be used in composite formulations with wheat flour for bread reformulation.

Bread is a commonly consumed food across the globe [18]. Thus, bread could be targeted as an essential delivery medium for bioactive compounds and essential nutrients present in plant-based food by-products [19-21]. There is an increased acceptability and demand from consumers for bread with beneficial health effect beyond normal nutritional nourishment. Bread with added value is referred to as "functional bread" [22]. Some beneficial health effects of functional bread beyond nutrition, including the attenuation of glycaemic response [20], improvements in satiety [23], reduction of abdominal visceral fat concentrations [24], and increased production of short chain fatty acids in the large intestine $[20,25,26]$, have been reported. The incorporation of plant-based food by-products in bread is a viable way of enhancing the nutritional value of the bread [27] which may reduce food waste and improve the nutritional status of consumers. That notwithstanding, the addition of plant-based food by-products as functional ingredients in bread influences consumers organoleptic perceptions [28] and has impacts on the bioactive properties and nutritional profile of bread [4,27]. In this review, the bioactivity of plant-based food by-products, the physical qualities, sensory and nutritional profiles of breads containing plant-based food by-products, and the bioavailability/bioaccessibility of the bioactive compounds in humans leading to potential health effects following bread consumption, as investigated through clinical trials, are reported.

\section{Bioactive Properties of Plant-Based Food By-Products from Industrial and Small-Scale Processing}

Plant-based food by-products contain a high concentration of bioactive compounds including phenolics [29,30]. Earlier comprehensive reviews on the classification, synthesis, and properties of phenolics have been reported [30,31]. Briefly, phenolics are classified into groups that include phenolic acids, flavonoids, tannins, stilbenes, lignins, and coumarins [31] and as secondary plant metabolites, they provide a self-defence role for the plants as they help the plants ward off foreign invaders [32]. Harsh environmental factors have been found to impact on the development of bioactive compounds in plants [32]. For example, plants that grow at high mountainous areas, drought areas, and in high salinity conditions naturally develop a high concentration of bioactive compounds compared to their counterparts that grow under normal conditions [32]. Bioactive compounds including polyphenols [33,34] demonstrate health effects such as impairing the onset of oxidative stress [35], reducing inflammatory markers [36], demonstrating antidiabetic properties through glycaemic response attenuation [33,35], and exhibiting cardiovascular protective effects [35] demonstrated through in vitro and human clinical trials. Consequently, they can potentially promote health and improve the well-being of consumers following their consumption. The bioactive properties of plant-based food by-products, including rice bran, wheat bran, corn bran, oat bran, rootlets, brewers spent grain, fruits pomaces, and artichoke stem/leaves, have been reported in this review. 
Rice bran is a by-product of rice kernel dry milling and consists largely of the germ, pericarp, aleurone and sub-aleurone layers, and oil [37]. It is rich in dietary fibre, essential fatty acids, plant sterols $\gamma$-oryzanol, tocopherols, and tocotrienols [11], and biologically active peptides derived from its protein [38], all of which have potential health effects. The predominant bioactive compound in the bran of rice, $\gamma$-oryzanol is postulated to demonstrate antioxidant, hypocholesterolemic, anti-cancer, and anti-diabetic capabilities [11]. Pang et al. [39] analysed the phenolic and antioxidant potential of the bran component of white, red, and black rice cultivars. The authors reported that the bran fractions of the different rice cultivars contain antioxidant compounds with the concentration of bound total phenolic acid being significantly higher than the free counterpart in the bran [39]. Phenolics, based on their ability to exist freely or bound to other macromolecules, impacts on their classification as either insoluble-bound phenolics, or free, or esterified forms [31]. For the bound phenolics in rice cultivars, which most of the time are present in higher concentration [39], Shahidi and Yeo [31] reported that, due to their impaired absorption in the small intestine, they transit into the large intestine where they are fermented through microbial activities resulting in the degradation of the cell wall matrix. This consequently leads to increased phenolic release that affects physiological processes and demonstrate health effects including anticancer, anti-inflammation and improving cardiovascular support [31]. It is worthwhile to note that the cultivar type of rice influences the phenolic composition and antioxidant potential of the bran [39]. Enzymatic hydrolysates and peptides extracted from rice bran proteins have been shown to demonstrate significant health effects including antioxidant, antidiabetic, anti-cancer, and mitigating against the action of angiotensin converting enzyme which is involved in the pathogenesis of hypertension [38]. However, advances made in the area of bioactive peptides research is in the early phase and have been carried out mostly using in vitro and animal models [40]. More investigations in the form of human clinical trials are warranted to validate the health effects of bioactive peptides [41].

Wheat bran constitutes about $14.5 \%$ of the wheat kernel [42]. However, its use in bread is constrained due to its adverse impacts on the textural properties of bread which is attributed to the ability of its fibre to dilute gluten [1], consequently making bread crumb harder. Nevertheless, there is some evidence to show that this challenge could be overcome when the bran is subjected to pre-treatment processes including fermentation [43,44]. Wheat bran is a rich source of essential bioactive compounds. The concentration of total phenolics and flavonoids in wheat bran have been reported to be in the range of 430.6-844.7 mg gallic acid equivalents (GAE)/100 $\mathrm{g}$ and 160.6-330.7 mg catechin equivalence (CE)/100 g dry weight respectively whilst the antioxidant activity ranged from 37.5 to $50.9 \%$ radical scavenging activity (RSA) using 2,2-diphenyl-1-picrylhydrazyl (DPPH) assay [45]. De Camargo et al. [30] reported that enzymes involved in the biosynthesis of bioactive compounds in plants are tissue specific. Consequently, the concentration of bioactives synthesised in the bran of wheat could vary from that synthesised in the endosperm. For pigmented wheat varieties, bran fraction from yellow, purple, and blue wheat types have been reported to contain a higher concentration of phenolic acids, anthocyanins, dietary fibre, and $\beta$-glucans, thus increasing total antioxidant activity compared to their refined flour counterparts [46]. Furthermore, the bran of wheat contains proteins that are of higher nutritional quality, rich in bioactive peptides [47], and contain a significant amount (12.8 $\mathrm{mg} / 100 \mathrm{~g}$ dry weight) of tocochromanols [48] which could be exploited for new food product development.

Corn is another important cereal grain that demonstrates antioxidant potential and consumed in several countries across the globe. Dry milling of dried corn results in the generation of the bran. Corn bran contains a high concentration of tocochromanols [48] which refers to tocopherol and tocotrienol homologues in a collective sense [49]. It must be emphasized that tocopherol and tocotrienol are antioxidant vitamins [50] and thus are ideal candidate in impairing free radical propagation reactions that leads to oxidative stress. The bran of corn contains higher concentrations of insoluble dietary fibre composed primarily of cellulose, which consists of approximately $280 \mathrm{~g} / \mathrm{kg}$, and hemicellulose constituting approximately $700 \mathrm{~g} / \mathrm{kg}$ [51]. Potential health effects associated with the consumption of food enriched with dietary fibre include binding to bile acids and their subsequent release through the 
faeces, fermentation and release of organic acids including propionate, preventing the rapid absorption of glucose and laxation enhancement [52]. Consequently, it has been reported that health-conscious bread consumers are willing to eat bread enriched with fibre in Poland [53]. Corn bran is a promising ingredient for the bread industry, however the correct proportion of it must be established when used in recipe formulation to deliver bread that will not only demonstrate health effects, but also acceptable organoleptic attributes for consumers. In addition to the rich fibre content, corn bran has total phenolic and ferulic acid concentration ranging from 1538.0-1925.0 mg GAE/100 $\mathrm{g}$ and 228.0-624.10 mg CE/100 g respectively on dry weight basis [45] and antioxidant activity ranging from $40.30-71.30 \%$ RSA with DPPH assay [45].

Oat processing generates bran as by-products which are a rich source of bioactive compounds. Polyphenolic and flavonoid compounds have been shown to abound in oat bran extract compared to wholegrain endosperm extracts [54]. Additionally, oat bran of Avon and Sargodha-81 varieties were reported to contain $\beta$-glucan concentrations of $5.59 \%$ and $4.63 \%$ respectively [55] and demonstrates effective free radical scavenging abilities [54]. The challenge associated with oat bran utilisation in bread is that the physical and organoleptic properties of bread are negatively impacted due to the weakening of the gluten network when used in increased proportions with wheat flour for bread formulation.

As food industry by-product, rootlets could be a valuable addition to the bioactive profile of functional bread. Rootlets contain total phenol contents of $102 \mathrm{mg} / \mathrm{L}$ [13]. The beer processing industry carries out malting on barley, resulting in the generation of rootlets [13] which are usually discarded or fed to animals. In a similar vein, brewer's spent grain has been reported to contain bioactive compounds that have health-promoting benefits. It has polyphenols, insoluble fibre, arabinoxylan and total fibre contents of $131.0 \mathrm{mg} / \mathrm{L}, 48.35 \%, 22.24 \%$, and $48.22 \%$ (on $w / w$ ) respectively and contains $0.26 \%$ $(w / w)$ gamma-aminobutyric acid [14]. The need to explore incorporating these by-products into bread is justified.

Fruits and vegetables are highly perishable due to their high moisture and water activity [56]. There is therefore the need to process them into powders and other ready-to-use end product to make them available off-season and take advantage of low cost during seasonal period. Fruits and vegetable processing results in the generation of by-products that are rich in bioactives [30]. For example, the antioxidant activity of dietary fibre prepared from mango peels at a concentration of $0.05 \%$ has been reported to be stronger than that of 2-tertbutyl-4-hydroxyanisol (BHA) and French PARAD'OX (a commercially sold polyphenol concentrate) and of DL- $\alpha$-tocopherol respectively [57]. Lycopene is a bioactive compound effective in ameliorating the progression of prostate cancer in men [58]. Dried tomato by-product (skin and seed) produced from tomato juice processing has been reported to have lycopene and $\beta$-carotene contents of 174.12 and $32.66 \mathrm{mg} / \mathrm{Kg}$ respectively [17]. Additionally, the authors reported that the total phenolic and antioxidant activity were $865.77 \mathrm{mg} \mathrm{GAE} / \mathrm{kg}$ and $2.46 \mathrm{mmol}$ Trolox $/ \mathrm{kg}$, respectively [17]. The prospect of utilising tomato seeds in bread remains a viable option to improve the nutritional and bioactive profile of the bread. Other researchers have added seeds, including watermelon seeds [59], flaxseed, and sunflower seeds [60] to bread. Shyu et al. [15] pre-processed fermented and unfermented citrus peels using different dry hot-air temperatures $\left(100^{\circ} \mathrm{C}\right.$ and $150{ }^{\circ} \mathrm{C}$ ) and evaluated the bioactive properties of the powders produced. The unfermented citrus peel extract, particularly processed at $150^{\circ} \mathrm{C}$, demonstrated increased antioxidant activity and contained total polyphenols and flavonoids, including nobiletin and tangeretin [15], which are likely due to the destruction and changes that impacted on cell wall polysaccharides and other matrix factors during the thermal treatment $\left(150^{\circ} \mathrm{C}\right)$, resulting in an increased extractability of phenolic compounds [61]. Skenderidis et al. [62] freeze-dried, milled and assayed bioactive properties of pomegranate peels. The authors reported a total polyphenol content of $79.46 \mathrm{mgGAE} / \mathrm{g}$ extract (DW) for pomegranate powder. In that study, pomegranate peels demonstrated antimicrobial properties against certain pathogenic and spoilage microorganisms, including E. coli, S. aureus, S. typhimurium, C. perfringens, C. jejuni, L. monocytogenes, M. fructicola, and F. oxysporum, which are responsible for 
foodborne challenges. There is potential for pomegranate peel to militate against food spoilage caused by microorganisms and oxidation.

Pomaces from two varieties of grape ("Zelen" and "Merlot") were dried, milled into flour and their bioactivity evaluated [2]. The authors reported total phenolic content of 28.29 and $32.73 \mathrm{mg}$ GAE/g dw and antioxidant activity using DPPH of 89.31 and $88.30 \mathrm{mg}$ GAE/g dw for the "Zelen" and "Merlot" varieties of grape respectively [2]. This demonstrates that, the bioactive composition of grape pomace varies depending on cultivar type.

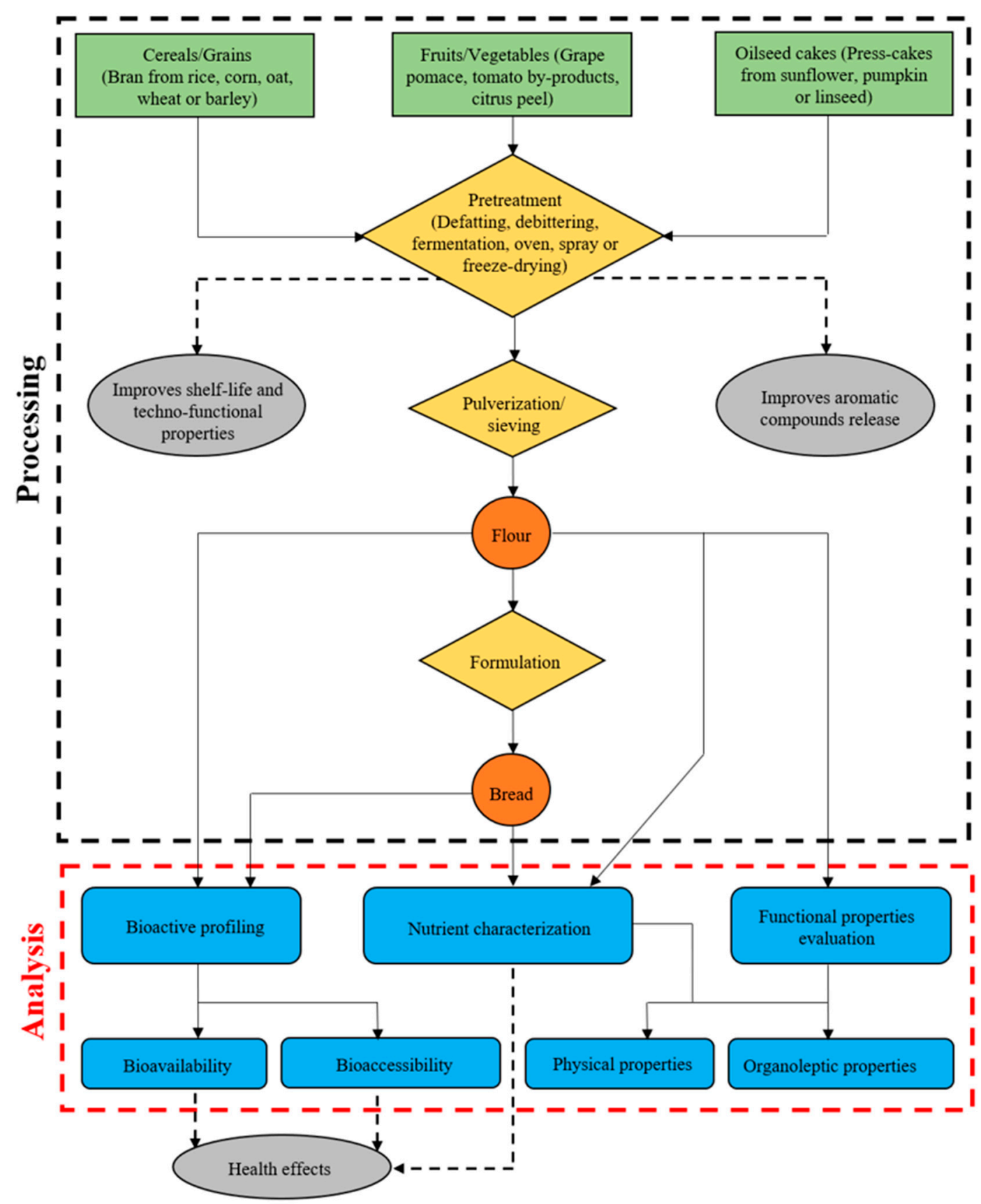

Figure 1. A flow chart showing the processes involved in the valorisation of plant-based food by-products for bread development and the validation of the health effects.

The distribution of phenolics in plants varies from one plant part to the other [63] as enzymes involved in phenolic biosynthesis are tissue specific [30]. For example, analyses of total phenolics and 
cynaropicrin in the leaves and stems of artichoke which are by-products from artichoke processing showed a wide variation when compared to the head [63]. Total polyphenol contents of 8.8, 21.6, and $34.7 \mathrm{mg} \mathrm{GAE} / \mathrm{g}$ DW and total cynaropicrin content of $0.014,20.8$, and $2.7 \mathrm{mg} / \mathrm{g}$ DW has been reported for the head, leaves, and steam of the artichoke plant [63]. This highlights the need to utilise artichoke leaves and stems which are mainly discarded during processing of artichoke head in the development of new food products including bread.

The review of literature shows that plant-based food by-products contain high concentrations of bioactive compounds and demonstrate antioxidant properties. Consequently, their utilisation in bread formulation to promote health effects is therefore justifiable. That notwithstanding, the need to investigate the effect on the qualities, sensory and nutritional profiles of bread following plant-based food by-products enrichment is warranted. A flow chart showing the processes involved in the valorisation of plant-based food by-products for bread development and the validation of the health effects is shown below (Figure 1).

\section{Qualities of Functional Bread Containing Plant-Based By-Products}

Evaluation of the qualities of bread is important because it impacts on the organoleptic properties of the bread and includes loaf weight, specific volume, textural attributes, and colour $[27,64]$. The determination of loaf volume for example is essential in predicting the specific volume which provides insight into the performance of processes involved in the bread-making [65]. The qualities of bread tend to be influenced by the ingredients used in the bread formulation and the baking parameters including temperature and time used during baking.

Currently, to the best of our knowledge, no study on bread has standardized consumers' priorities in terms of bread selection with reference to the qualities (specific volume, color, hardness, chewiness, gumminess, and cohesiveness). However, certain essential elements are given consideration by the baker and the consumer. For example, to the baker, bread with lower specific volume may have lower returns on the financial gains of the bakery. Bread color can attract consumers and reveal the baking conditions, including baking temperature and time to the baker. Crumb hardness is essential to the consumer as it impacts on the overall mastication feel in the mouth. Thus, three topmost factors that will be focused on for the selection of the proportion of functional ingredient used in bread with favourable substitution would be specific volume, hardness of the crumb, and bread color (Table 1).

\subsection{Fruit and Vegetable By-Product Valorization in Functional Bread}

Struck et al. (2018), while studying how wheat macromolecules, namely glutenin and gliadin, interact with blackcurrant berry pomace in a dough, found that the development of gluten is impaired following incorporation of the pomace resulting in a dough with lower stickiness, resistance and extensibility. Exploring further at the microscopic level, the authors observed disruption of gluten network due to the pomace fibre incorporation. Consequently, bread enrichment with fibre from lemon pomace (0-9\%) [67], apple, pear, and date pomaces [74], and pineapple pomace (at 10\% substitution) [4] resulted in increased crumb hardness $[4,67]$ and reduced specific volume $\left(4.60,4.30\right.$ and $3.0 \mathrm{~cm}^{3} / \mathrm{g}$ for the "control", $5 \%$ and 10\% pineapple-fibre enriched bread; 2.9 for control and $2.7 / 2.8 \mathrm{~cm}^{3} / \mathrm{g}$ for the date, pear, and apple pomace fibre-enriched breads, respectively) [4,74]. Additionally, mango peel fibre (at 1\%,3\% and 5\%) [66] and fibre-rich flour from artichoke by-product (3\%,6\%, $9 \%$, and $12 \%$ ) [16] supplementation in bread resulted in a general decrease in loaf and specific volume $[16,66]$ whereas crumb moisture and loaf density increased as the substitution increased [66], as shown in (Table 1). Fibres from plant-based by-products vary in their ability to hold water in dough matrix with some types having good water holding capacity whereas others are poor [85]. Although, fibre weakens the gluten network in bread dough, fibre with many hydrophilic compounds, and consequently having good water-retaining properties, could produce bread with better crumb properties than their poor water-retention counterparts. Pectin, for example, is one known hydrophilic fibre with a high water retaining property [86]. 
Table 1. Plant-based food by-products valorisation in functional bread formulation and their impact on bread qualities.

\begin{tabular}{|c|c|c|c|c|c|c|}
\hline Functional Ingredients & $\begin{array}{l}\text { Substitution } \\
\text { Levels }\end{array}$ & $\begin{array}{c}\text { Treatment of Plant-Based } \\
\text { by-Products }\end{array}$ & $\begin{array}{c}\text { Favourable } \\
\text { Substitution Level }\end{array}$ & $\begin{array}{c}\text { Effects on Qualities of } \\
\text { Bread }\end{array}$ & $\begin{array}{l}\text { Quantitative Variations from the } \\
\text { Control Measures }\end{array}$ & References \\
\hline & \multicolumn{6}{|c|}{ Vegetables and fruit by-products utilization } \\
\hline Tomato by-product & 6 and $10 \%$ & drying at $60^{\circ} \mathrm{C}$ & N/A & $\downarrow$ specific volume. & $\begin{array}{c}6 \% \text { substitution-specific volume } \\
\downarrow(6.6 \%), 10 \% \text { substitution-specific } \\
\text { volume } \downarrow(7.1 \%)\end{array}$ & [17] \\
\hline $\begin{array}{c}\text { Artichoke } \\
\text { (Cynarascolymus L.) } \\
\text { by-product }\end{array}$ & $3,6,9$ and $12 \%$ & lyophilized and milled & $3 \%$ & $\begin{array}{l}\downarrow \text { loaf volume except for } \\
\text { the } 3 \% \text { substitution, } \\
\uparrow \text { crumb hardness and } \\
\downarrow \text { crumb L* values }\end{array}$ & $\begin{array}{c}3 \% \text { substitution-specific volume }(0 \%), \\
\text { crumb hardness } \uparrow(27.2 \%), \mathrm{L}^{*} \downarrow(12.8 \%), \\
6 \% \text { substitution-specific volume } \\
\downarrow(\sim 8 \%), \text { crumb hardness } \uparrow(39.9 \%), \mathrm{L}^{*} \\
\downarrow(31.1 \%), 9 \% \text { substitution-specific } \\
\text { volume } \downarrow(\sim 8 \%), \text { crumb hardness } \\
\uparrow(58.9 \%), \mathrm{L}^{*} \downarrow(36 \%) \text { and } 12 \% \\
\text { substitution-specific volume } \downarrow(\sim 32 \%), \\
\text { crumb hardness } \uparrow(78 \%) \text { and } \mathrm{L}^{*} \downarrow(41.5 \%) \\
\end{array}$ & [16] \\
\hline Raw mango peel powder & 1,3 and $5 \%$ & $\begin{array}{l}\text { blanching, wet milling, } \\
\text { pulping, drying and } \\
\text { milling }\end{array}$ & & $\begin{array}{l}\downarrow \text { specific volume and } \\
\text { whiteness index. } \uparrow \text { loaf } \\
\text { density }\end{array}$ & $\begin{array}{c}1 \% \text { substitution-specific volume } \\
\downarrow(9.7 \%), \text { whiteness index } \downarrow(5 \%), \text { loaf } \\
\text { density } \uparrow(9.8 \%), 3 \% \\
\text { substitution-specific volume } \downarrow(17.1 \%) \text {, } \\
\text { whiteness index } \downarrow(8.9 \%) \text { and loaf } \\
\text { density } \uparrow(16 \%), 5 \% \text { substitution-specific } \\
\text { volume } \downarrow(21.9 \%) \text {, whiteness index } \\
(12 \%) \text { and loaf density } \uparrow(23 \%) \text {. }\end{array}$ & [66] \\
\hline Lemon pomace fibre & 3,6 and $9 \%$ & N/A & $3 \%$ & $\begin{array}{l}\downarrow \text { specific volume and } \\
\uparrow \text { crumb hardness }\end{array}$ & $\begin{array}{c}3 \% \text { substitution- specific volume } \\
\downarrow(\sim 33 \%), \text { crumb hardness } \uparrow(\sim 50 \%), 6 \% \\
\text { substitution- specific volume } \downarrow(\sim 44 \%), \\
\text { crumb hardness } \uparrow(\sim 68.8 \%) \text { and } 9 \% \\
\text { substitution- specific volume } \downarrow(\sim 50 \%), \\
\text { crumb hardness } \uparrow(\sim 81.8 \%) .\end{array}$ & [67] \\
\hline Pineapple pomace fibre & 5 and $10 \%$ & N/A & $5 \%$ & $\begin{array}{c}\downarrow \text { specific volume. } \uparrow \text { crumb } \\
\text { hardness except for } 5 \% \\
\text { substitution. } \downarrow \text { crumb L* } \\
\text { values. }\end{array}$ & $\begin{array}{c}5 \% \text { substitution-specific volume } \\
\downarrow(6.5 \%), \text { crumb hardness } \downarrow(10.9 \%), \\
\text { crumb L* } \downarrow \text { ( } 2 \%) \text { and } 10 \% \\
\text { substitution-specific volume } \downarrow(34.8 \%) \text {, } \\
\text { crumb hardness }(57.3 \%) \text { and crumb L* } \\
\downarrow(3.6 \%) .\end{array}$ & [4] \\
\hline
\end{tabular}


Table 1. Cont.

\begin{tabular}{|c|c|c|c|c|c|c|}
\hline Functional Ingredients & $\begin{array}{l}\text { Substitution } \\
\text { Levels }\end{array}$ & $\begin{array}{c}\text { Treatment of Plant-Based } \\
\text { by-Products }\end{array}$ & $\begin{array}{c}\text { Favourable } \\
\text { Substitution Level }\end{array}$ & $\begin{array}{c}\text { Effects on Qualities of } \\
\text { Bread }\end{array}$ & $\begin{array}{l}\text { Quantitative Variations from the } \\
\text { Control Measures }\end{array}$ & References \\
\hline $\begin{array}{l}\text { Grape pomace flour } \\
\text { from Merlot and Zelen } \\
\text { cultivars }\end{array}$ & 6,10 and $15 \%$ & drying and milling & $6 \%$ & $\begin{array}{c}\downarrow \text { specific volume, } \uparrow \text { crumb } \\
\text { hardness except for the } 3 \% \\
\text { Merlot, Zelen and } 10 \% \\
\text { Zelen grape pomace flour } \\
\text { substitution and } \downarrow L^{*} \\
\text { values }\end{array}$ & $\begin{array}{c}\text { 6\% substitution (Merlot grape)-specific } \\
\text { volume } \downarrow(\sim 7.1 \% \%), \text { hardness } \downarrow(\sim 12.3), \\
L^{*} \downarrow(35.5 \%), \text { (Zelen grape)-specific } \\
\text { volume } \downarrow(\sim 7.1 \%), \text { crumb hardness } \\
\downarrow(\sim 29 \%), L^{*} \downarrow(29.2 \%), 10 \% \text { substitution } \\
\text { (Merlot grape)-specific volume } \downarrow(\sim 9 \%), \\
\text { crumb hardness } \uparrow(11.5 \%), L^{*} \downarrow(40.3 \%) \text {, } \\
\text { (Zelen grape)-specific volume }(\sim 9 \%), \\
\text { crumb hardness } \downarrow(\sim 5.8 \%), L^{*}(33.3 \%) \text {, } \\
15 \% \text { substitution }(\text { Merlot grape)-specific } \\
\text { volume } \downarrow(\sim 8.6), \text { crumb hardness } \\
\uparrow(\sim 18.8 \%), L^{*} \downarrow(46.7 \%),(\text { Zelen } \\
\text { grape)-specific volume } \downarrow(\sim 17.8 \%), \\
\text { crumb hardness }(\sim 1.4 \%), L^{*}(\sim 37.5 \%) \text {. }\end{array}$ & [2] \\
\hline Grape seed flour (GSF) & $\begin{array}{c}2.5,5,7.5 \text {, and } 10 \mathrm{~g} \\
\text { GSF/ } 100 \mathrm{~g} \text { wheat } \\
\text { flour }\end{array}$ & N/A & $2.5-5 \%$ & $\begin{array}{c}\downarrow \text { loaf volume, } \uparrow \text { crumb } \\
\text { hardness and } \downarrow \text { loaf } \\
\text { brightness }\end{array}$ & $\begin{array}{c}2.5 \mathrm{~g} \text { GSF/100 g wheat flour } \\
\text { substitution-loaf volume } \downarrow(7 \%) \text {, crumb } \\
\text { hardness } \uparrow(7.6 \%), 5 \mathrm{~g} \text { GSF } / 100 \mathrm{~g} \text { wheat } \\
\text { flour substitution-loaf volume } \downarrow(12.8 \%), \\
\text { crumb hardness } \uparrow(14.2 \%), 7.5 \mathrm{~g} \text { GSF/100 } \\
\mathrm{g} \text { wheat flour substitution-loaf volume } \\
\downarrow(18.4 \%), \text { crumb hardness } \uparrow(34.8 \%), 10 \\
\mathrm{~g} \mathrm{GSF} / 100 \mathrm{~g} \text { wheat flour } \\
\text { substitution-loaf volume }(26.1 \%) \text { and } \\
\text { crumb hardness } \uparrow(51.4 \%)\end{array}$ & {$[68]$} \\
\hline Grape seed extract & $\begin{array}{l}300 \mathrm{mg}, 600 \mathrm{mg} \\
\text { and } 1 \mathrm{~g}\end{array}$ & N/A & $\begin{array}{c}300 \mathrm{mg}, 600 \mathrm{mg} \text { and } \\
1 \mathrm{~g}\end{array}$ & $\begin{array}{l}\uparrow c r u m b \text { hardness with the } \\
\text { exception of the } 300 \\
\text { mg-substituted bread. } \downarrow L^{*} \\
\text { values }\end{array}$ & $\begin{array}{c}300 \mathrm{mg} \text { substitution-crumb hardness } \\
\downarrow(\sim 3.75), \mathrm{L}^{*} \downarrow(8.8 \%), 600 \mathrm{mg} \\
\text { substitution-crumb hardness } \downarrow(1.2 \%), \\
\mathrm{L}^{*} \downarrow(12 \%) \text {, and } 1 \mathrm{~g} \text { substitution } \downarrow(1.2 \%) \\
\text { and } \mathrm{L}^{*} \downarrow(12.9 \%)\end{array}$ & [69] \\
\hline Pomegranate seed flour & $5,7.5$ and $10 \%$ & drying and milling & & $\begin{array}{l}\downarrow \text { loaf volume and } L^{*} \\
\text { values. } \uparrow \text { hardness }\end{array}$ & $\begin{array}{c}5 \% \text { substitution- loaf volume } \downarrow(21.9 \%), \\
\mathrm{L}^{*} \downarrow(23.7 \%), \text { crumb hardness } \uparrow(35.8 \%), \\
7.5 \% \text { substitution- loaf volume } \\
\downarrow(29.6 \%), \mathrm{L}^{*} \downarrow(27.2 \%), \text { crumb hardness } \\
\uparrow(50 \%) \text { and } 10 \% \text { substitution-loaf } \\
\text { volume } \downarrow(36.4 \%), \mathrm{L}^{*} \downarrow(30.3 \%), \text { crumb } \\
\text { hardness } \uparrow(53.1 \%) .\end{array}$ & [70] \\
\hline
\end{tabular}


Table 1. Cont.

\begin{tabular}{|c|c|c|c|c|c|c|}
\hline Functional Ingredients & $\begin{array}{l}\text { Substitution } \\
\text { Levels }\end{array}$ & $\begin{array}{c}\text { Treatment of Plant-Based } \\
\text { by-Products }\end{array}$ & $\begin{array}{c}\text { Favourable } \\
\text { Substitution Level }\end{array}$ & $\begin{array}{c}\text { Effects on Qualities of } \\
\text { Bread }\end{array}$ & $\begin{array}{c}\text { Quantitative Variations from the } \\
\text { Control Measures }\end{array}$ & References \\
\hline $\begin{array}{l}\text { Kiwifruit polyphenol } \\
\text { extract (KPE), } \\
\text { blackcurrant polyphenol } \\
\text { extract (BPE) and apple } \\
\text { polyphenol extract (APE) } \\
\text { with high methoxy } \\
\text { pectin (HM) }\end{array}$ & $\begin{array}{c}(3 \% \mathrm{HM} \\
\text { pectin+3\% KPE), } \\
(3 \% \mathrm{HM} \\
\text { pectin+3\% BPE }) \\
\text { and ( } 3 \% \mathrm{HM} \\
\text { pectin+3\% APE) }\end{array}$ & N/A & & $\begin{array}{c}\downarrow \text { loaf volume and } \uparrow c r u m b \\
\text { hardness }\end{array}$ & $\begin{array}{c}\text { (3\% HM pectin+3\% KPE) } \\
\text { substitution-specific volume } \downarrow(23.5 \%) \text {, } \\
\text { crumb hardness } \uparrow(26.7 \%),(3 \% \text { HM } \\
\text { pectin+3\% BPE) substitution- specific } \\
\text { volume } \downarrow(12.1 \%) \text {, crumb hardness } \\
\uparrow(35.3 \%) \text { and ( } 3 \% \text { HM pectin }+3 \% \text { APE) } \\
\text { substitution- specific volume } \downarrow(26.3 \%) \\
\text { and crumb hardness } \uparrow(71.4 \%) .\end{array}$ & [71] \\
\hline Apple pectin & $\begin{array}{l}\text { Apple pectin(s) of } \\
\text { low molecular } \\
\text { weight and high } \\
\text { molecular weight } \\
\text { at a ratio of } 1: 1 \text {, at } \\
\text { concentrations of } \\
3 \text { or } 6 \% w / w) \text {, in } \\
\text { the absence or } \\
\text { presence of added } \\
\text { kiwifruit phenolic } \\
\text { extract, apple } \\
\text { phenolic extract or } \\
\text { blackcurrant } \\
\text { phenolic extract } \\
\text { (at } 3 \% w / w) .\end{array}$ & 3 and $6 \%$ & $3 \%$ & $\begin{array}{l}\downarrow \text { loaf volume and } \mathrm{L}^{*} \\
\text { values }\end{array}$ & $\begin{array}{c}3 \% \text { apple pectin substitution-loaf } \\
\text { volume } \downarrow(2.2 \%), L^{*} \downarrow(9.2 \%), 6 \% \text { apple } \\
\text { pectin substitution- loaf volume } \downarrow(24 \%), \\
L^{*} \downarrow(12.2 \%), 3 \% \text { pectin and } 3 \% \text { kiwifruit } \\
\text { phenolic extract substitution-loaf } \\
\text { volume } \downarrow(28 \%), L^{*} \downarrow(18.6), 3 \% \text { apple } \\
\text { pectin and } 3 \% \text { apple phenolic extract } \\
\text { substitution-loaf volume } \downarrow(33.5 \%), L^{*} \\
\downarrow(21.3 \%) \text { and } 3 \% \text { apple pectin and } 3 \% \\
\text { blackcurrant phenolic extract } \\
\text { substitution-loaf volume } \downarrow(12 \%) \text { and } L^{*} \\
\downarrow(31.8 \%)\end{array}$ & [72] \\
\hline $\begin{array}{l}\text { Orange extract powder } \\
(\mathrm{OE}) \text {, pomegranate } \\
\text { extract powder (PE), } \\
\text { elderberry extract } \\
\text { powder (EE) and yeast } \\
\text { extract powder (YE) }\end{array}$ & $\begin{array}{l}4 \% \mathrm{EE}, 36 \% \mathrm{EE}, 4 \% \\
\mathrm{OE}, 8 \% \mathrm{OE}, 4 \% \mathrm{PE}, \\
16 \% \mathrm{PE} \text { and } 4 \% \mathrm{YE}\end{array}$ & $\begin{array}{c}\mathrm{OE}=\text { Hot water blanching } \\
\text { of orange peel, oven } \\
\text { drying, ethanol extraction, } \\
\text { oven drying and milling. } \\
\mathrm{PE}=\text { Hot water blanching, } \\
\text { oven drying and milling. } \\
\mathrm{EE}=\text { Ethanol extraction, } \\
\text { oven drying and milling } \\
\mathrm{YE}=\text { Autolysis, Base and } \\
\text { acid extractions, } \\
\text { lyophilizing and milling }\end{array}$ & $4 \%$ & $\begin{array}{c}\uparrow \text { loaf volume for } 4 \% \text { EE, } \\
4 \% \text { OE and } 4 \% \text { YE. Crumb } \\
\text { hardness for } 4 \% \text { EE, } 36 \% \\
\text { EE, } 4 \% \text { OE and } 4 \% \text { YE } \\
\text { showed no significant } \\
\text { difference compared to the } \\
\text { control but was } \\
\text { significantly } \downarrow \text { for } 4 \% \text { EE }\end{array}$ & $\begin{array}{c}4 \% \text { elderberry extract } \\
\text { substitution-specific volume } \uparrow(15.8 \%) \text {, } \\
\text { crumb hardness } \downarrow(40.4 \%), 36 \% \\
\text { substitution- specific volume } \downarrow(16.6 \%) \text {, } \\
\text { crumb hardness } \uparrow(9 \%) \text {, orange extract: } \\
4 \% \text { substitution-specific volume } \\
\uparrow(9.7 \%) \text {, crumb hardness } \downarrow(15.5 \%), 8 \% \\
\text { substitution-specific volume }(10.2 \%) \text {, } \\
\text { pomegranate extract: } 4 \% \text { substitution- } \\
\text { specific volume } \downarrow(9.1 \%) \text {, crumb } \\
\text { hardness } \uparrow(20.9 \%), 16 \% \\
\text { substitution-specific volume } \downarrow(37.4 \%) \text {, } \\
\text { crumb hardness } \uparrow(69 \%) \text {, yeast extract: } \\
4 \% \text { substitution-specific volume }(1.1 \%) \\
\text { and crumb hardness } \downarrow(4.5 \%) \text {. }\end{array}$ & [73] \\
\hline
\end{tabular}


Table 1. Cont.

\begin{tabular}{|c|c|c|c|c|c|c|}
\hline Functional Ingredients & $\begin{array}{l}\text { Substitution } \\
\text { Levels }\end{array}$ & $\begin{array}{c}\text { Treatment of Plant-Based } \\
\text { by-Products }\end{array}$ & $\begin{array}{c}\text { Favourable } \\
\text { Substitution Level }\end{array}$ & $\begin{array}{c}\text { Effects on Qualities of } \\
\text { Bread }\end{array}$ & $\begin{array}{l}\text { Quantitative Variations from the } \\
\text { Control Measures }\end{array}$ & References \\
\hline \multirow[t]{2}{*}{$\begin{array}{l}\text { Flesh fibre concentrate } \\
\text { from apple, pear, and } \\
\text { date pomaces }\end{array}$} & $2 \%$ of fibre & cooked fruit by-products & $2 \%$ & $\begin{array}{l}\text { favourable effect on } \\
\text { specific volume of bread } \\
\text { loaves and on the crumb } \\
\text { and crust texture }\end{array}$ & $\begin{array}{c}\text { Apple fibre: } 2 \% \text { substitution-specific } \\
\text { volume } \downarrow(3.4 \%) \text {, pear fibre: } 2 \% \\
\text { substitution- specific volume } \downarrow(6.9 \%) \\
\text { and date fibre: } 2 \% \text { substitution-specific } \\
\text { volume } \downarrow(6.9 \%) \text {. }\end{array}$ & [74] \\
\hline & \multicolumn{6}{|c|}{ Grains/cereals and pseudocereal by-products } \\
\hline $\begin{array}{l}\text { Processed full-fat (FFRB) } \\
\text { and defatted (DFRB) } \\
\text { bran from long, medium } \\
\text { and short grain }\end{array}$ & 10 and $20 \%$ & $\begin{array}{l}\text { freezing rice bran, } \\
\text { autoclave stabilization, } \\
\text { drying, ricebran slurry } \\
\text { preparation, drum-drying } \\
\text { and defatting. }\end{array}$ & $10 \%$ FFRB & $\begin{array}{l}\downarrow \text { specific volume with the } \\
\text { exception of the } 10 \% \text { FFRB } \\
\text { from long grain. } \uparrow \text { crumb } \\
\text { hardness for DFRB bread } \\
\text { than for FFRB. }\end{array}$ & $\begin{array}{c}\text { Full fat rice bran- } 10 \% \text { substitution: long } \\
\text { grain rice bran- specific volume } \uparrow(1.3 \%) \text {, } \\
\text { medium grain rice bran- specific } \\
\text { volume } \downarrow(0.2 \%) \text {, short grain rice } \\
\text { bran-specific volume } \downarrow(1.1 \%) \text {, Defatted } \\
\text { rice bran- } 10 \% \text { substitution-long grain } \\
\text { rice bran-specific volume } \downarrow(10.7 \%) \text {, } \\
\text { medium grain rice bran- specific } \\
\text { volume } \downarrow(3.9 \%) \text {, short grain rice } \\
\text { bran-specific volume } \downarrow(5.7 \%) \text {, Full fat } \\
\text { rice bran- } 20 \% \text { substitution-long grain } \\
\text { rice bran- specific volume } \downarrow(7.9 \%) \text {, } \\
\text { medium grain rice bran-specific } \\
\text { volume } \downarrow(4.6 \%) \text {, short grain rice bran- } \\
\text { specific volume } \downarrow(13.4 \%) \text {, defatted rice } \\
\text { bran- } 20 \% \text { substitution-long grain rice } \\
\text { bran- specific volume } \downarrow(18.6 \%) \text {, } \\
\text { medium grain rice bran- specific } \\
\text { volume } \downarrow(16.2 \%) \text {, short grain rice } \\
\text { bran-specific volume } \downarrow(19.7 \%) \text {. }\end{array}$ & [75] \\
\hline $\begin{array}{l}\text { Dietary fibre from } \\
\text { defatted rice bran }\end{array}$ & 5 and $10 \%$ & $\begin{array}{l}\text { defatting, gelatinization, } \\
\text { digestion with protease, } \\
\text { incubation with } \\
\text { amyloglucosidase, } \\
\text { precipitation of soluble } \\
\text { dietary fibre using alcohol, } \\
\text { filtration and oven-drying }\end{array}$ & & $\begin{array}{c}\uparrow c r u m b \text { hardness and } \\
\downarrow \text { loaf volume }\end{array}$ & $\begin{array}{c}5 \% \text { substitution-loaf volume } \downarrow(22.9 \%), \\
\text { crumb hardness } \uparrow(47.6 \%) \text { and } 10 \% \\
\text { substitution-loaf volume } \downarrow(37.8 \%), \\
\text { hardness } \uparrow(79.6 \%) .\end{array}$ & [76] \\
\hline Rice bran & $10 \%$ & $\begin{array}{l}\text { fermentation using } L \text {. } \\
\text { plantarum, } L \text {. mesenteroides } \\
\text { and } L . \text { delbrueckii }\end{array}$ & $\begin{array}{l}10 \% \text { with } L \text {. } \\
\text { mesenteroides }\end{array}$ & $\begin{array}{l}\downarrow \text { crumb hardness for } \\
\text { sourdough breads. } \uparrow \text { loaf } \\
\text { volume for bread from } \\
\text { sourdoughs fermented by } \\
\text { L. mesenteroides }\end{array}$ & N/A & [77] \\
\hline
\end{tabular}


Table 1. Cont

\begin{tabular}{|c|c|c|c|c|c|c|}
\hline Functional Ingredients & $\begin{array}{l}\text { Substitution } \\
\text { Levels }\end{array}$ & $\begin{array}{c}\text { Treatment of Plant-Based } \\
\text { by-Products }\end{array}$ & $\begin{array}{c}\text { Favourable } \\
\text { Substitution Level }\end{array}$ & $\begin{array}{c}\text { Effects on Qualities of } \\
\text { Bread }\end{array}$ & $\begin{array}{l}\text { Quantitative Variations from the } \\
\text { Control Measures }\end{array}$ & References \\
\hline $\begin{array}{l}\text { Middling fraction (M) of } \\
\text { wholegrain (WM) and } \\
\text { pearled (PM) barley }\end{array}$ & $15,30,45$ and $60 \%$ & pearling & $\begin{array}{c}30 \% \mathrm{WM} \text { and } 15 \% \\
\text { PM }\end{array}$ & $\begin{array}{c}\text { loaf volume not affected } \\
\text { for } 30 \% \text { WM and } 15 \% \\
\text { PM-substituted breads } \\
\text { significantly. } \uparrow \text { hardness for } \\
\text { barley bread }\end{array}$ & $\begin{array}{c}15 \% \text { barley middlings-loaf } \\
\text { volume-whole } \downarrow(\sim 4.1 \%) \text { and pearled } \\
\downarrow(\sim 4.8 \%), 30 \% \text { middlings-loaf } \\
\text { volume-whole } \downarrow(6.2 \%) \text { and pearled } \\
\downarrow(\sim 11.56 \%), 45 \% \text { middlings-loaf } \\
\text { volume-whole } \downarrow(\sim 17 \%) \text { and pearled } \\
\downarrow(\sim 25.2 \%), 60 \% \text { middlings-loaf volume- } \\
\text { whole } \downarrow(\sim 31.5 \%) \text { and pearled }(\sim 31.5 \%) \text {. }\end{array}$ & [78] \\
\hline Oat bran & 10,20 and $30 \%$ & $\begin{array}{l}\text { enzymatic bioprocessing } \\
\text { with xylanase and } \\
\text { sourdough fermentation }\end{array}$ & & $\begin{array}{l}\text { xylanase and sourdough } \\
\text { addition increased the } \\
\text { bread specific volume and } \\
\text { reduced crumb hardness }\end{array}$ & $\begin{array}{c}\text { Oat bran: } 10 \% \text { substitution-specific } \\
\text { volume } \downarrow(\sim 3.4 \%) \text {, crumb hardness } \\
\uparrow(\sim 10 \%), 20 \% \text { substitution-specific } \\
\text { volume } \downarrow(\sim 13.8 \%) \text {, crumb hardness } \\
\uparrow(25.6 \%), 30 \% \text { substitution-specific } \\
\text { volume } \downarrow(\sim 24.1 \%), \text { crumb hardness } \\
\uparrow(\sim 35.7 \%) \text {, sourdough + oat bran, } 10 \% \\
\text { substitution-specific volume }(\sim 8.6 \%), \\
\text { crumb hardness } \uparrow(\sim 25 \%), 20 \% \\
\text { substitution-specific volume } \downarrow(\sim 16.7 \%) \text {, } \\
\text { crumb hardness }(\sim 5.6 \%), 30 \% \\
\text { substitution-specific volume } \downarrow(\sim 14.3 \%) \\
\text { and crumb hardness } \uparrow(\sim 55.6 \%) .\end{array}$ & [79] \\
\hline Wheat bran & $\begin{array}{c}\text { Sequential } \\
\text { pearling of wheat } \\
\text { kernels to } 3,6,9 \\
\text { and } 12 \% \text { by } \\
\text { weight }\end{array}$ & $\begin{array}{l}\text { thermal treatment and } \\
\text { native }\end{array}$ & & $\begin{array}{c}\downarrow \text { bread volume and } \\
\uparrow \text { crumb hardness }\end{array}$ & $\begin{array}{c}\text { Ground bran-hardness } \uparrow(55.2 \%) \text {, } \\
\text { pearlings, } 0-3 \% \text {, crumb hardness } \\
\uparrow(70.9 \%) \text {, heat-treated pearlings, } 0-3 \% \text {, } \\
\text { crumb hardness } \uparrow(65 \%) \text {, pearlings, } \\
3-6 \% \text {, crumb hardness } \uparrow(64.4 \%) \text {, } \\
\text { pearlings, } 6-9 \% \text {, crumb hardness } \\
\uparrow(52.6 \%), \text { pearlings, } 6-12 \% \text {, crumb } \\
\text { hardness } \uparrow(49 \%)\end{array}$ & [80] \\
\hline $\begin{array}{l}\text { Wheat bran and germ } \\
\text { mixture }\end{array}$ & $\begin{array}{l}15 \%(w / w) \text { of } \\
\text { fermented (and } \\
\text { unfermented) } \\
\text { milling } \\
\text { by-products }\end{array}$ & fermentation & $15 \%$ & $\begin{array}{c}\uparrow \text { specific volume and } \\
\downarrow \text { crumb hardness }\end{array}$ & $\begin{array}{c}\text { Specific volume } \uparrow(8.9) \text { and crumb } \\
\text { hardness } \downarrow(15.8 \%)\end{array}$ & [43] \\
\hline
\end{tabular}


Table 1. Cont

\begin{tabular}{|c|c|c|c|c|c|c|}
\hline Functional Ingredients & $\begin{array}{l}\text { Substitution } \\
\text { Levels }\end{array}$ & $\begin{array}{c}\text { Treatment of Plant-Based } \\
\text { by-Products }\end{array}$ & $\begin{array}{c}\text { Favourable } \\
\text { Substitution Level }\end{array}$ & $\begin{array}{c}\text { Effects on Qualities of } \\
\text { Bread }\end{array}$ & $\begin{array}{l}\text { Quantitative Variations from the } \\
\text { Control Measures }\end{array}$ & References \\
\hline $\begin{array}{l}\text { Buckwheat (Fagopyrum } \\
\text { Esculentum Moench) bran }\end{array}$ & $20 \%$ & $\begin{array}{c}\text { enzymatic treatment with } \\
\text { transglutaminase (TG) and } \\
\text { sodium stearoyl-2-lactylate } \\
\text { (SSL) }\end{array}$ & $\begin{array}{l}20 \% \text { with SSL + TG } \\
(0.5 \%+0.4 \%)\end{array}$ & $\begin{array}{c}\downarrow \text { bread volume for } \\
\text { buckwheat bran bread. } \\
\text { Combination of SSL + TG } \\
\text { to the bran significantly } \\
\text { improved bread crumb } \\
\text { lightness, volume and } \\
\text { crumb softness }\end{array}$ & $\begin{array}{l}\text { Buckwheat bran ( } 20 \% \text { substitution } \\
\text { without any additive)-specific volume } \\
\downarrow(38.3 \%) \text {, crumb hardness } \uparrow(51 \%), \\
\text { crumb L* }(32.1 \%)\end{array}$ & [81] \\
\hline Rootlets & $5,10,15$ and $20 \%$ & fermentation & $5 \%$ & $\begin{array}{c}\downarrow \text { specific volume except } \\
\text { the } 5 \% \text { substitution and } \\
\uparrow \text { crumb hardness }\end{array}$ & $\begin{array}{c}5 \% \text { substitution-specific volume } \\
\uparrow(6.9 \%), \text { crumb hardness } \uparrow(34 \%), 10 \% \\
\text { substitution- specific volume } \uparrow(9 \%), \\
\text { crumb hardness } \uparrow(52.7 \%), 15 \% \\
\text { substitution-specific volume } \downarrow(21.5 \%), \\
\text { crumb hardness } \uparrow(67.5 \%) \text { and } 20 \% \\
\text { substitution-specific volume } \downarrow(31.3 \%), \\
\text { crumb hardness } \uparrow(68.8 \%)\end{array}$ & [13] \\
\hline Brewers spent grain & $5,10,15$ and $20 \%$ & $\begin{array}{c}\text { fermentation using } \\
\text { Lactobacillus plantarum FST } \\
1.7\end{array}$ & $5 \%$ & $\begin{array}{l}\downarrow \text { specific volume. } \uparrow \text { crumb } \\
\text { hardness with the } \\
\text { exception of } 5 \% \text { fermented } \\
\text { brewers spent grain }\end{array}$ & $\begin{array}{c}5 \% \text { substitution-specific volume } \\
\downarrow(8.7 \%) \text {, crumb hardness } \downarrow(16.1 \%), 10 \% \\
\text { substitution-specific volume } \downarrow(12.6 \%) \text {, } \\
\text { crumb hardness } \uparrow(28.7 \%), 15 \% \\
\text { substitution-specific volume } \downarrow(22.4 \%) \text {, } \\
\text { crumb hardness } \uparrow(56.4 \%), 20 \% \\
\text { substitution-specific volume } \downarrow(28 \%) \\
\text { and crumb hardness } \uparrow(69.5 \%)\end{array}$ & [14] \\
\hline \multirow[t]{2}{*}{ Nejayote solids } & 3,6 and $9 \%$ & $\begin{array}{l}\text { vacuum filtration, freezing } \\
\text { and lyophilizing }\end{array}$ & 3 and $6 \%$ & $\begin{array}{c}\downarrow \text { loaf volume and } \\
\downarrow \text { whiteness }\left(\mathrm{L}^{*}\right) \text { values. }\end{array}$ & $\begin{array}{c}3 \% \text { substitution-loaf volume } \downarrow(2 \%), \mathrm{L}^{*} \\
\downarrow(2.5 \%), 6 \% \text { substitution-loaf volume } \\
\downarrow(5.1 \%), \mathrm{L}^{*} \downarrow(6.5 \%), \text { and } 9 \% \\
\text { substitution-loaf volume } \downarrow(9.5 \%), \mathrm{L}^{*} \\
\downarrow(7.4)\end{array}$ & [82] \\
\hline & \multicolumn{6}{|c|}{ Oilseed and bean by-products } \\
\hline $\begin{array}{l}\text { Naked pumpkin seed } \\
(\mathrm{PuC}) \text {, sunflower seed } \\
\text { (SC), yellow linseed (LC), } \\
\text { and walnut (WnC) cakes }\end{array}$ & 5 and $10 \%$ & N/A & & $\begin{array}{c}\uparrow \text { dough stability with } \\
\text { walnut cake enrichment } \\
\text { WnC }\end{array}$ & N/A & [8] \\
\hline
\end{tabular}


Table 1. Cont.

\begin{tabular}{|c|c|c|c|c|c|c|}
\hline Functional Ingredients & $\begin{array}{l}\text { Substitution } \\
\text { Levels }\end{array}$ & $\begin{array}{c}\text { Treatment of Plant-Based } \\
\text { by-Products }\end{array}$ & $\begin{array}{c}\text { Favourable } \\
\text { Substitution Level }\end{array}$ & $\begin{array}{c}\text { Effects on Qualities of } \\
\text { Bread }\end{array}$ & $\begin{array}{l}\text { Quantitative Variations from the } \\
\text { Control Measures }\end{array}$ & References \\
\hline Hemp seed cake & 5,10 and $20 \%$ & N/A & 5 and $10 \%$ & $\begin{array}{l}\downarrow \text { specific volume, } \uparrow \text { crumb } \\
\text { hardness and } \downarrow b^{*} \text { values }\end{array}$ & $\begin{array}{c}\text { 5\% substitution-specific volume } \downarrow(18 \%) \text {, } \\
\text { hardness } \uparrow(31.2) \text { and crumb } b^{*} \text { value } \\
\downarrow(12.8), 10 \% \text { substitution, specific } \\
\text { volume } \downarrow(41 \%) \text {, hardness } \uparrow(56 \%) \text { and } \\
\text { crumb } b^{*} \text { value } \downarrow(22.5 \%) \text { and } 20 \% \\
\text { substitution, specific volume } \downarrow(45.9 \%), \\
\text { hardness } \uparrow(71.3 \%) \text { and crumb } b^{*} \\
\downarrow(39.9 \%)\end{array}$ & [83] \\
\hline Flaxseed flour and marc & $5,10,15 \%$ & $\begin{array}{l}\text { cakes from cold pressed } \\
\text { flaxseed, milling and } \\
\text { pulverisation }\end{array}$ & $5 \%$ & $\downarrow$ specific volume & $\begin{array}{c}5 \% \text { substitution-flaxseed flour- specific } \\
\text { volume } \downarrow(6.3 \%) \text { and crumb hardness } \\
\uparrow(6.8 \%) \text {, flaxseed marc- specific volume } \\
\downarrow(4.6 \%) \text {, crumb hardness } \downarrow(2.4 \%), 10 \% \\
\text { substitution- flaxseed flour- specific } \\
\text { volume } \downarrow(13 \%) \text { and crumb hardness } \\
\uparrow(23 \%) \text {, flaxseed marc- specific volume } \\
\downarrow(13 \%) \text {, crumb hardness } \downarrow(2.4 \%) \text { and } \\
15 \% \text { substitution-flaxseed flour- specific } \\
\text { volume } \downarrow(18.6 \%) \text {, crumb hardness } \\
\uparrow(53.4 \%), \text { flaxseed marc- specific } \\
\text { volume } \downarrow(18.2 \%), \text { crumb hardness } \\
\uparrow(12.8 \%)\end{array}$ & [7] \\
\hline Cupuassu peel flour & $\begin{array}{l}3,6 \text {, and } 9 \% \\
\text { cupuassu peel } \\
\text { flour }\end{array}$ & $\begin{array}{l}\text { separation of peel from } \\
\text { pulp and seeds, cleaning in } \\
\text { hypochlorite solution, } \\
\text { freeze-drying and milling } \\
\text { into powder. }\end{array}$ & N/A & $\begin{array}{c}\uparrow b^{*} \text { values of the bread } \\
\text { crumb }\end{array}$ & $\begin{array}{c}3 \% \text { substitution- }-b^{*} \uparrow(23.6 \%) \\
6 \% \text { substitution- } b^{*} \text { value }(25.7 \%)-\text { and } \\
\left.9 \% \text { substitution- } b^{*} \text { value }(29.9 \%)\right)\end{array}$ & [84] \\
\hline
\end{tabular}

Note. $\uparrow=$ higher and $\downarrow=$ lower. $\mathrm{L}^{*}=$ Lightness, $\mathrm{a}^{*}$ —redness and $\mathrm{b}^{*}$ —yellowness. N/A—Not applicable 
Sporin et al. [2] reported that the cultivar type from which dried grape pomace flour is obtained impacts on the properties of bread dough and its crumb properties including colour, hardness and cohesiveness. This could be attributed to the different genetic composition of the different cultivars which may impact on their chemical and morphological properties. For instance, 'Merlot' grape pomace flour (GPF) with substitution at $6 \%, 10 \%$ and $15 \%$ in bread had a greater adverse effect on the crumb hardness of bread compared to the 'Zelen' GPF. Though grape flour addition in bread generally reduced the loaf volume of the supplemented breads, the addition of $6 \%$ 'Zelen' GPF to the bread significantly reduced the bread hardness [2]. A decrease in loaf brightness and volume with an increase in crumb hardness and porosity was recorded when grape seed flour (GSF) (replacement above $5 \mathrm{~g}$ GSF/100 g hard red spring wheat flour) was used in bread formulation [68]. Peng et al. [69] however reported that, except for the bread colour, adding grape seed extract to bread had a non-observable effect on its hardness.

The incorporation of pomegranate seed flour ( $5 \%, 7.5 \%$, and $10 \%$ levels) into wheat flour decreased loaf brightness, while increases were detected in loaf colour $\mathrm{a}^{*}$ and $\mathrm{b}^{*}$ values [70]. Martins et al. [73] incorporated orange extract powder, pomegranate extract powder, elderberry extract and yeast extract powder into bread and observed that there was a reduction in $L^{*}$ values in the darker crumbs when compared to the "control" bread [73]. Fibre-rich flour from artichoke by-products incorporated into wheat bread at substitutions $(3 \%, 6 \%, 9 \%$, and $12 \%)$ increased bread darkness with a resultant decrease in the lightness $L^{*}$ values as percentage substitution increased [16]. The changes in the cellular make-up of plant-based food by-products initiated by heat during baking results in the release of extractable bioactive compounds and other biomolecules, including proteins and sugars, which possibly promotes an increased Maillard browning process resulting in the generally decreasing trend of $L^{*}$ values observed for the breads formulated with plant-based food by-product ingredients [61].

Fruit and vegetable processing also generate by-products which are rich sources of pectin (polysaccharide mainly from the cell wall of the plant) and bioactive compounds including polyphenols [87]. Sivam et al. (2011) reported that the addition of fruits and its components/extracts (kiwifruit, apple and blackcurrant), polyphenol extract, and pectin (high and low mexthoxy content) to bread modified bread crumb hardness, specific volume, amount of free thiol groups of bread proteins, extractability of polyphenols and proteins from bread [71], and impacted the cross-linking of the dough microstructure and bread properties [72]. Bread enrichment with pectin and fruit phenolic extracts increased the water content (38.0-40.0\%) and bread weight (854.4-879.3 g), whereas bread volume decreased compared to the "control" bread devoid of extract additives. The authors thus postulated that, pectin and/or phenolic compounds interact with bread components such as wheat proteins during dough development and bread baking. This influences the cross-linking microstructure that occurs in the dough of bread and consequently impacts the properties of bread [72].

Composite flour formulated from wheat and tomato by-products (skin and seed) flour produced bread with water content of $26.41 \%, 28.15 \%$, and $29.83 \%$, titratable acidity (TA) of $0.008 \%, 0.010 \%$, and $0.012 \%$, as citric acid, specific volume of $5.18 \%, 4.84 \%$, and $4.81 \%$, and bread crumb porosity of $85.28 \%$, $84.24 \%$ and $84.15 \%$ for the "control", $6 \%$, and $10 \%$ tomato by-product substitution, respectively [17]. The relatively higher water content of the bread formulated with the tomato by-products could be due to the presence of hydrophilic fibre in it which have a high water absorption capacity. The challenge is that bread with high moisture content is susceptible to microbial growth, especially moulds [88]. Nevertheless, we have reported that, bread enriched with functional ingredients, tend to have a longer shelf-life than bread formulated from refined wheat flour [89]. This is due to the reduced oxidation of lipids and protein by the bioactives present inherently in the functional ingredients and the bioactives acting as antimicrobial agents against the growth of mould [89]. Broccoli by-products from the stem and leaves were incorporated into bread and its impact on bread qualities evaluated [6]. The incorporation of $2 \%(w / w)$ of broccoli by-products consisting of stem and leaves into bread resulted in significant reduction in specific volume of 2.77 and $2.81 \mathrm{~mL} / \mathrm{g}$ respectively compared to the control that recorded $2.91 \mathrm{~mL} / \mathrm{g}$. Interestingly, no significant difference was recorded in the loaf weight of the control $(48.10 \mathrm{~g})$ 
and the broccoli stem enriched breads $(47.67 \mathrm{~g})$ but not for the broccoli leaf-enriched bread $(49.26 \mathrm{~g})$. The authors reported that the potential antimicrobial agents in the broccoli by-products impaired yeast activity, resulting in reduced leavening and a lower specific volume [6].

\subsection{Cereals/Grains/Pseudo-Cereal Industrial By-Products Valorisation in Functional Bread}

In the case of fibre from cereal/grain and pseudo-cereal by-products, Han et al. [90] reported an increasing hardness of wheat bran dietary fibre (WBDF)-enriched doughs and attributed this to the weakening gluten protein aggregation and the gradual formation of intermolecular hydrogen bonds between gluten and starch. The authors reported that, for bran-enriched flour products, pre-treatment leading to size reduction, softening, or solubility is warranted to improve dough properties and performance.

Lima et al. [75] incorporated processed full-fat (FFRB) and defatted (DFRB) bran from three rice cultivars (long, medium, and short grain) into bread and reported its effect on the textural properties. The supplementation of wheat flour with rice bran caused an (increase in loaf volume for the FFRB and a decrease for DFRB for $10 \%$ substitute) and (a decrease for FFRB and DFRB for loaf volume for $20 \%$ substitute). Bran from the medium rice grain elicited the highest loaf volume. The hardness, gumminess and chewiness of bread increased with elevated levels of rice bran and was higher for DFRB bread than for FFRB. The formulation of bread using $10 \%$ FFRB showed no adverse impact on the bread's textural properties but a slight hardening of the bread was recorded for the loaves formulated with $20 \%$ FFRB, when compared to the control. In a comparable manner, the supplementation of bread with 5 and $10 \%$ dietary fibre preparations derived from defatted rice bran significantly reduced loaf volume and increased bread crumb hardness [76]. However, when Farahmand et al. [77] fermented rice bran through sourdough preparation using L. plantarum, L. mesenteroides, and L. delbrueckii, the authors observed that the "control" bread had a significantly higher crumb hardness compared to the rice bran sourdough breads with $L$. mesenteroides-fermented dough recording a significantly higher loaf volume compared to the "control" bread.

Sullivan et al. [78] formulated bread using "middling" fraction (M) of wholegrain (WM) and pearled (PM) barley $(15 \%, 30 \%, 45 \%$, and $60 \%$ substitution) with wheat flour and reported that, the loaf volume was not significantly affected up to $30 \% \mathrm{WM}$ and $15 \%$ PM substitution. Bread crumb hardness increased as levels of barley flour substitution intensified (Sullivan et al. 2011). Xylanase and sourdough fermentation of dough formulated with oat bran modified the soluble and insoluble fibres and consequently increased specific volume and reduced crumb hardness [79] which were desirable properties of the bread. Eventually, when the addition of $10 \%, 20 \%$, and $30 \%$ oat bran was used in wheat flour substitution, a significant increase in dough development time and weakening was observed [79], possibly due to gluten dilution.

Hemdane et al. [80] in their quest to gain insight into the causes of bread volume and crumb texture quality loss following the incorporation of wheat bran, studied the hydration properties of wheat bran and bran-associated enzyme activities of ground and pearled bran fractions, and related them to their impact on bread making. The pericarp-rich fraction recorded the highest water absorption and retention capacity whereas the aleurone-rich fraction recorded lower. In terms of the bread qualities, the pericarp-rich fraction adversely impacted bread volume and crumb texture compared to the aleurone-rich fractions. In comparing a heat treated and a native pericarp-rich fraction having a high bran-associated enzymatic activity, it was revealed that bran-associated enzymes impacted on the crumb textural properties but not bread volume [80]. Bread formulated with use of fermented wheat bran and germ mixture recorded a higher specific volume $\left(2.24 \mathrm{~cm}^{3} / \mathrm{g}\right)$ and lower hardness $(2947 \mathrm{~g})$ compared to the unfermented $\left(1.91 \mathrm{~cm}^{3} / \mathrm{g}\right.$ and $\left.3700 \mathrm{~g}\right)$ and refined white wheat bread $\left(0.21 \mathrm{~cm}^{3} / \mathrm{g}\right.$ and $3500 \mathrm{~g}$ ) counterparts. The bread formulated with the use of fermented wheat bran and germ had $\mathrm{L}^{*}, \mathrm{a}^{*}$, and $\mathrm{b}^{*}$ values (46.1, 14.7 and 28.7) compared to the unfermented (52.3, 12.5, and 30.1) and refined white bread (65.1, 7.1, and 31.9) respectively [43]. Fermentation thus promises to be an 
effective pre-treatment process that could be exploited to improve the techno-functional properties of plant-based food by-products including wheat bran.

The impact of buckwheat (Fagopyrum esculentum Moench) bran, transglutaminase (TG) and sodium stearoyl-2-lactylate (SSL) on bread properties has been reported. Buckwheat bran-enriched bread had reduced loaf volume and lightness (L) values of crust and crumb. Interestingly, the combination of SSL + TG to the bran significantly improved loaf volume, crumb softness (for three days) and $L^{*}$ values of the bread [81]. Brewers spent grain and fermented brewers spent grain $(5 \%, 10 \%, 15 \%$, and $20 \%)$ using Lactobacillus plantarum FST 1.7 incorporated into bread reduced specific volume with the volume reducing as the substitution increased when compared to the "control" wheat bread. Bread formulated with fermented brewers spent grain $(5 \%)$ had a softer crumb and lower chewiness textural attributes compared to control breads formulated with refined wheat and wholemeal wheat flour [14].

The supplementation of bread with nejayote solids (3\%, $6 \%$, and $9 \%)$, a by-product from corn processing, resulted in a reduction in $\mathrm{L}^{*}$ values with significant differences existing between controls and the substituted breads with the exception of the $3 \%$ nejayote-supplemented bread. It was shown that, as nejayote solid substitution in bread increased, bread weight increased whereas bread volume decreased. The was no significant difference between the "control" bread and the nejayote solid substituted bread in terms of the textural attributes (cohesiveness, hardness, chewiness, and elasticity) analysed with the texture analyser [82].

Wheat bread enrichment with rootlets (at 5\%,10\%, 15\%, and 20\% levels), a malting by-product, and its qualities has been studied and compared to two control breads (refined wheat and wholemeal flour). The $5 \%$ substituted breadcrumbs were significantly softer than the control bread formulated with wholemeal wheat flour. The chewiness of the bread generally increased with increasing addition of rootlets [13].

\subsection{Oilseed and Seed by-Products Valorisation for Functional Bread Application}

The pressing of oil seeds for oils results in the generation of cake as a by-product. Oilseed cake obtained from sunflower seed, walnut, naked pumpkin seed, and yellow linseed have been used in bread formulation (at $5 \%$ and $10 \%$ substitution) and their impact on wheat flour dough reported [8]. Generally, a reduced dough elasticity, increased water absorption and dough development time attributed largely to the enhanced fibre and protein content was observed. Dough stability, however, increased with walnut cake substitution and decreased with pumpkin or sunflower cake flour substitution [8]. This reveals that different seed cakes have different physicochemical properties which impacts on bread dough properties. Flaxseed by-products, including flaxseed flour and marc, were incorporated into bread at $5 \%, 10 \%$, and $15 \%$, and its effect on bread qualities was investigated [7]. There was generally a significant decrease in the specific volume of all the flaxseed flour $\left(2.67,2.48,2.32 \mathrm{~cm}^{3} / \mathrm{g}\right.$ for $5 \%, 10 \%$, and $15 \%$ substitutions respectively) and marc-enriched breads $\left(2.48\right.$ and $2.33 \mathrm{~cm}^{3} / \mathrm{g}$ for $10 \%$ and $15 \%$ substitutions respectively) compared to the control sample $\left(2.85 \mathrm{~cm}^{3} / \mathrm{g}\right)$, apart from the $5 \%$ flax marc incorporated bread $\left(2.72 \mathrm{~cm}^{3} / \mathrm{g}\right)$. The crumb moisture content of all flaxseed flour $(42$, $45.1,45.9 \mathrm{~g} / 100 \mathrm{~g})$ and marc enriched breads $(42,44.7$ and $45.9 \mathrm{~g} / 100 \mathrm{~g})$ was significantly higher than their corresponding control counterpart $(39.6 \mathrm{~g} / 100 \mathrm{~g})$. This property of the flax by-product-enriched breads to maintain crumb moisture could be attributed to the presence of hydrophilic macromolecules including mucilage and gums in flaxseed [91,92]. Thus, they can bind to water during the dough preparation and improve bread keeping qualities. Hardness of bread crumb was not significantly differently from the control bread apart from the $10 \%$ and $15 \%$ flax marc-enriched breads.

Hemp seed cake by-product was used to substitute wheat flour at proportions $5 \%, 10 \%$, and $20 \%$ and the impacts on bread properties studied. At up to $10 \%$ hemp substitution, dough strength and stability were not negatively impacted. However, an adverse effect was observed when substitution increased to $20 \%$ [83]. The use of cupuassu peel flour, a by-product of cupuassu beans processing, in bread formulation has also been reported. The specific volume of bread reduced following cupuassu peel flour $3 \%, 6 \%$, and $9 \%$ substitution with conventional wheat flour. In terms of bread crumbs, the $L^{*}$ 
values were higher for the samples with $3 \%$ cupuassu peel flour, followed by the sample with $6 \%$ cupuassu peel flour and the control sample. Bread crumb of the cupuassu-substituted breads recorded more yellowish colour compared to the "control" [84].

In summary, the addition of plant-based food by-products generally adversely impact on the loaf volume and crumb hardness especially with increasing substitution. There is therefore a need to explore more pre-treatment methods that could be applied to plant-based food by-products to improve the qualities of bread when they are incorporated. The need to consider the appropriate amount of flour from the food by-product for bread formulation is imperative to produce bread with desirable qualities.

\section{Bioactivity of Functional Bread Formulated with Food Processing By-Products}

The bioactive properties of functional bread are impacted by the bioactive compounds including phenolic compounds and carotenoids present in the functional ingredients used for the bread formulation. The presence of these compounds in bread is essential as they favourably extend the shelf-life properties by reducing the oxidation of lipids and proteins and inhibiting microbial growth [89].

\subsection{Fruit and Vegetable By-Products Utilisation in Bread and Effect on Bioactive Properties}

The cell walls of fruit and vegetable by-products are rich sources of bioactive compounds [30]. Total phenolic content and DPPH scavenging properties ranging from (443-794 mg GAE/100 gm) and (33.61-68.54\% inhibition) was recorded for $1 \%, 3 \%$, and $5 \%$ raw mango peel powder-substituted breads respectively compared to the "control" bread that had 227.66 GAE/100 gm and 21.51\% inhibition. Consequently, the $5 \%$ substitution recorded the highest level of bioactivity [66].

Bread enrichment with peel powder from pomegranate peel powder [93], grape seed flour [68], grape seed extract [69], and lemon pomace fibre [67] enhanced the total phenolic, antioxidant content, and DPPH radical scavenging abilities of the bread.

Sporin et al. [2] reported that the properties of bread fortified with grape pomace flour (6, 10 and $15 \%$ ) is dependent on the varietal type used. Bread with the highest grape pomace flour supplementation (15\%) recorded the highest total phenolic contents of $5.92 \mathrm{mg}$ gallic acid equivalents (GAE)/g dw for 'Merlot' and $3.65 \mathrm{mg}$ gallic acid equivalents/g dw for 'Zelen' respectively. The highest antioxidant activity was reported for the 'Merlot' grape pomace flour (15\%) supplemented bread [2]. Peng et al. [69] reported that the formation of $\mathrm{N} \varepsilon$-(carboxymethyl) lysine, an advanced glycation end-product that adversely affects human health, was found to be impaired in bread when bread was enriched with the grape seed extract. Generally, the bioactivity of the supplemented breads increased as the concentration of the raw mango peel powder substitution increased. Artichoke stem incorporated into bread digested under simulated duodenal and colonic phases revealed total phenolics and flavonoids concentration (745 and $25.6 \mu \mathrm{g} / \mathrm{g}$ DW), (1696.3 and $48.5 \mu \mathrm{g} / \mathrm{g} \mathrm{DW})$ and $(2414.2$ and $64.9 \mu \mathrm{g} / \mathrm{g} \mathrm{DW}$ ) respectively for 3\%, $6 \%$, and $9 \%$ artichoke stem incorporated breads [63].

\subsection{Grain/Cereal, Pseudocereal By-Products Utilisation in Bread and Effect on Bioactive Properties}

Wheat milling by-products, wheat bran and germ mixture (ratio of 4:1), fermented using Lactobacillus plantarum DSM 32,248 and Lactobacillus rossiae DSM 32,249 have been used in bread formulation and compared with their unfermented and refined white wheat bread counterparts for their bioactivity [43]. Bread containing the fermented wheat bran and germ mixture recorded significantly higher concentration of total phenols and antioxidant activity compared to bread containing unfermented milling by-products and with refined white flour. Total phenols (29.0, 24.0 and $7.2 \mathrm{mmol} / \mathrm{kg})$, antioxidant activity $(65.00,58$ and $40 \mathrm{DPPH})$ and soluble fibre $(1.63 \%, 1.21 \%$, and $0.82 \%$ ) have been reported respectively for the bread with fermented, unfermented wheat bran and germ mixture and refined white wheat bread [43]. Dall'Asta et al. [94] investigated the bioaccessibility (the release) of phenolic acids from an aleurone-enriched bread and a whole grain bread from durum 
wheat and whole grain wheat flour using in-vitro digestion model. The authors reported that, the bioaccessibility of the phenolic acids in the aleurone-enriched bread was higher than in the whole grain bread highlighting the health-promoting potential of the aleurone-enriched bread. Bioprocessing of rye bran through enzymatic means and the application of sourdough fermentation on rye bran in functional bread formulation has been investigated. The degradation of the structural make-up of the rye bran particularly the cell wall, through the activities of enzymes, stimulated the release of phenolic compounds in the rye bread. This was prominent particularly in the rye bran treated with the enzyme mixture and with yeast fermentation [95]. Higher concentrations of phenolic acid were released from the bio-processed bran enriched-bread with ferulic acid consistently recording increased concentrations [96]. The concentration of total phenolic content of oat bran enriched bread has been observed to increase following the application of xylanase enzyme treatment and sourdough fermentation of the dough [79].

The use of nejayote solid from a wastewater obtained from lime-cooking of maize in functional bread formulation has been reported. In this study, wastewater from the lime-cooking was vacuum-filtered through a $280 \mathrm{~mm}$ filter. The solids retained were frozen, lyophilized and used in formulation $(3 \%, 6 \%$, and $9 \%$ ) with wheat. The $9 \%$ nejayote-enriched bread showed 745 times more ferulic acid compared to the "control" bread without nejoyote. Increasing the supplementation of nejayote $(3 \%, 6 \%$, and $9 \%)$ in bread impacted an increase proportionally in ferulic acid concentration. A similar trend was observed for the for the antioxidant capacity. Breads supplemented with $9 \%$ free extract nejoyote solid recorded an increase of $70 \%$ antioxidant capacity compared to the control bread (1.36-2.32 $\mu \mathrm{M}$ trolox/100 g). A significant difference existed in the antioxidant capacity of all the bound extract of nejayote-substituted bread compared to the control bread [82].

\subsection{Tuber Processing By-Products Utilisation and Effects in Bioactive Properties}

Sho et al. [97] enriched bread with sweet potato-derived shochu distillery by-product supernatants (SDBS) and reported that, the SDBS enriched-breads showed greater concentration of bioactive compounds including citric acid, gamma-aminobutyric acid, and polyphenolic components compared to the "control" bread. Consequently, significantly higher DPPH radical scavenging activity was demonstrated by the SDBS-enriched breads than the control bread, and this is attributed to the polyphenolic content [97].

\section{Sensory and Nutritional Profile of Functional Bread Formulated with Food By-Products}

Consumers are interested in bread with health-promoting properties yet organoleptic attributes that appeal to their perceptions is crucial towards bread liking [22]. There is a need to ensure that the correct substitution of plant-based food by-products is used in composite formulation with wheat flour to produce bread with acceptable organoleptic and enhanced nutritional properties. In regard to the bread choice with the favourable substitution of flour from plant-based food by-products, with particular reference to sensory profile, generally, the overall acceptability rating of the bread by consumers was prioritised (Table 2). 
Table 2. Plant-based food by-product utilisation in bread and their effect on sensorial and nutritional properties.

\begin{tabular}{|c|c|c|c|c|c|}
\hline Ingredients & Substitution Levels & Pre-Treatment & Findings & Acceptable Substitution & References \\
\hline \multicolumn{6}{|c|}{ Fruits and vegetables by-products } \\
\hline $\begin{array}{l}\text { Fermented and unfermented } \\
\text { citrus peels }\end{array}$ & 2,4 and $6 \%$ & $\begin{array}{c}\text { fermentation and thermal } \\
\text { treatment with hot air at } 100 \text { and } \\
150{ }^{\circ} \mathrm{C} \text {. }\end{array}$ & $\begin{array}{l}\uparrow \text { acceptability for unfermented citrus } \\
\text { peel flour-enriched bread }\end{array}$ & $\begin{array}{c}4 \text { and } 6 \% \text { unfermented citrus } \\
\text { peels treated at with hot dry } \\
\text { air at } 150 \text { and } 100{ }^{\circ} \mathrm{C} \\
\text { respectively }\end{array}$ & [15] \\
\hline Grape pomace flour & 6,10 and $15 \%$ & drying and milling & $\begin{array}{l}\uparrow \text { sand feeling in the mouth and was } \\
\text { affected by the variety of grape used }\end{array}$ & N/A-Descriptive test & [2] \\
\hline Grape seed flour & $\begin{array}{l}2.5,5,7.5 \text { and } 10 \mathrm{~g} \\
\text { GSF/100 } \mathrm{g} \text { wheat flour }\end{array}$ & N/A & $\begin{array}{l}\downarrow \text { ratings in astringency and sweetness } \\
\text { ( } \geq 7.5 \mathrm{~g} \text { GSF/100 gHRS) substitution). }\end{array}$ & $\begin{array}{l}\text { Up to } 5 \mathrm{~g} \text { GSF/100 g wheat } \\
\text { flour }\end{array}$ & [68] \\
\hline Lemon pomace fibre & 3,6 and $9 \%$ & N/A & $\begin{array}{l}\downarrow \text { sensory values for flavour, texture, } \\
\text { and overall acceptability ( } 6 \text { and } 9 \%) \text {. }\end{array}$ & $3 \%$ & [67] \\
\hline Pomegranate seed flour & $5,7.5$ and $10 \%$ & N/A & $\begin{array}{l}\uparrow \text { fibre content and appealing } \\
\text { organoleptic sensations for the } 5 \% \\
\text { substitution }\end{array}$ & $5 \%$ & [70] \\
\hline Pineapple pomace fibre (PPF) & 5 or $10 \%$ & N/A & $\downarrow$ sensory liking score (10\% substitution) & $5 \%$ & [4] \\
\hline \multicolumn{6}{|c|}{ Grains/cereals, pseudocereals by-products } \\
\hline $\begin{array}{l}\text { Wheat bran and germ } \\
\text { mixture }\end{array}$ & $15 \%(w / w)$ & fermentation & $\begin{array}{l}\text { } \text { pprotein scores, in-vitro protein } \\
\text { digestibility, essential amino acid index } \\
\text { and biological values (fermented wheat } \\
\text { bran and germ mixture). } \uparrow \text { dietary fibre } \\
\text { for enriched breads }\end{array}$ & N/A & [43] \\
\hline $\begin{array}{l}\text { Dietary fibre from defatted } \\
\text { rice bran }\end{array}$ & 5 and $10 \%$ & $\begin{array}{c}\text { defatting, gelatinization, } \\
\text { digestion with protease, } \\
\text { incubation with } \\
\text { amyloglucosidase, precipitation } \\
\text { of soluble dietary fibre using } \\
\text { alcohol, filtration and } \\
\text { oven-drying }\end{array}$ & $\uparrow$ higher fibre (5 and $10 \%$ rice bran) & N/A & [76] \\
\hline $\begin{array}{l}\text { “Middling" fraction (M) of } \\
\text { wholegrain (WM) and } \\
\text { pearled (PM) barley }\end{array}$ & $15 \%, 30 \%, 45 \%$ and $60 \%$ & pearling & $\begin{array}{l}\text { no significant difference was found in } \\
\text { the acceptability of WM and PM breads }\end{array}$ & Up to $30 \%$ barley middling & [78] \\
\hline $\begin{array}{c}\text { Buckwheat (Fagopyrum } \\
\text { Esculentum Moench) milling } \\
\text { products }\end{array}$ & $20 \%$ & $\begin{array}{l}\text { enzymatic treatment with } \\
\text { transglutaminase (TG) and } \\
\text { sodium stearoyl-2-lactylate (SSL) }\end{array}$ & $\begin{array}{l}\text { \overall acceptability score for } \\
\text { buckwheat bran-enriched bread. } \uparrow \text { fibre, } \\
\text { protein, ash, mineral and fat contents } \\
\text { for buckwheat bran enriched bread }\end{array}$ & & [81] \\
\hline
\end{tabular}


Table 2. Cont.

\begin{tabular}{|c|c|c|c|c|c|}
\hline Ingredients & Substitution Levels & Pre-Treatment & Findings & Acceptable Substitution & References \\
\hline Rootlets & $5 \%, 10 \%, 15 \%$ and $20 \%$ & fermentation & $\uparrow$ liking of organoleptic attributes & $5 \%$ fermented rootlet & [13] \\
\hline Brewers spent grain & $5,10,15$ and $20 \%$ & $\begin{array}{c}\text { fermentation using the lactic acid } \\
\text { bacteria, Lactobacillus plantarum } \\
\text { FST } 1.7\end{array}$ & $\begin{array}{l}\uparrow \text { liking for bread enriched with brewers } \\
\text { spent grain or fermented brewers spent } \\
\text { grain (using the lactic acid bacteria, } \\
\text { Lactobacillus plantarum FST 1.7) }\end{array}$ & $10 \%$ & [14] \\
\hline Nejayote solids & $3,6,9 \%$ & $\begin{array}{c}\text { vacuum filtration, freezing and } \\
\text { lyophilizing }\end{array}$ & $\begin{array}{l}\text { no adverse effect on the textural } \\
\text { perception by consumers. }\end{array}$ & Up to $9 \%$ & [82] \\
\hline \multicolumn{6}{|c|}{ Oilseed by-products } \\
\hline $\begin{array}{c}\text { Cake from from naked } \\
\text { pumpkin seed (PuC), } \\
\text { sunflower seed (SC), yellow } \\
\text { linseed (LC), and walnut } \\
\text { (WnC) }\end{array}$ & $5 \%$ and $10 \%$ & $\mathrm{~N} / \mathrm{A}$ & $\uparrow$ brown appearancefor WnC (walnut) & $5 \%$ SC & [8] \\
\hline Flaxseed flour and marc & 5,10 and $15 \%$ & milling and pulverization & $\uparrow$ protein, ash and total dietary fibre & $5 \%$ & [7] \\
\hline
\end{tabular}

Note. $\uparrow=$ higher and $\downarrow=$ lower. N/A—Not applicable. 


\subsection{Fruit and Vegetable By-Products Utilisation in Bread and Effects on Sensory Profile}

The incorporation of fruit by-products including fermented and unfermented citrus peels [15], lemon pomace fibre [67], raw mango peels [66], grape pomace flour and seed [2,68], and pineapple pomace fibre [4] impacted on the nutritional and sensorial properties of bread (Table 2). Evaluation of the organoleptic perceptions associated with raw mango powder incorporation into bread showed an increase in the intensity of hardness, rubbery texture, fruity aroma, after taste and traditional bread aroma [66]. The fruity aroma perceived for the mango-enriched bread could be due to the release of volatile aroma compounds during baking and possibly enhanced by Maillard reaction from the reaction of sugars from the mango peels with proteins from the wheat. Bread with unfermented citrus peel flour $(2 \%, 4 \%$, and $6 \%)$ recorded a greater acceptability compared to its fermented counterpart. Specifically, bread formulated with $4 \%$ and $6 \%$ unfermented citrus peels treated with hot dry air at $150{ }^{\circ} \mathrm{C}$ and $100{ }^{\circ} \mathrm{C}$, respectively, were significantly higher in terms of the overall sensory acceptability [15].

Grape pomace flour addition in bread affected the odour and taste intensity impacting onerous organoleptic sensations including increased intensity of aftertaste and gritty feeling in the mouth. The authors however noted that, the variety of grape pomace has a greater effect on the sensory attributes of the bread and "Zelen" variety was found to impact desirable attributes to consumers [2]. For flour from grape seed, bread with higher grape seed flour substitution ( $\geq 7.5 \mathrm{~g} \mathrm{GSF} / 100 \mathrm{gHRS})$ elicited lower ratings with respect to astringency and sweetness whereas formulation with up to $5 \%$ GSE/100 g flour had no significant adverse effect on the organoleptic perceptions of consumers [68]. Grape pomace and its seed contain phenolic compounds which are secondary plant metabolites, which though have health-promoting properties, are known for their bitter taste. In that regard, when utilising flour from grape pomace and seed in bread formulations, it is worthwhile to perform a preliminary investigation into what quantity of the flour when used in composite formulation with wheat flour will give bread that has favourable organoleptic attributes.

The addition of lemon pomace fibre $(6 \%$ and $9 \%)$ into bread resulted in significantly low sensory scores for the flavor, texture and overall acceptability. However, no significant difference was observed for bread with $3 \%$ lemon pomace fibre compared to those of the control bread. The authors concluded that bread formulated with 3\% lemon pomace fibre had sensorial attributes that resonated well with consumers organoleptic perceptions [67]. In a similar manner, the addition of pineapple pomace fibre at $10 \%$ to bread significantly impacted adversely on the sensory liking scores. The overall liking score of bread with $5 \%$ pineapple pomace fibre was, however, not significantly different from that of the control [4].

Flour from dried tomato by-product (skin and seed) enrichment with wheat resulted in bread with acceptable taste and overall liking for a $6 \%$ substitution but not for $10 \%$ substitution [17]. The lower acceptability score recorded for the $10 \%$ substituted bread could be attributed to the higher titratable acid score recorded as increased TA concentration relates to lower $\mathrm{pH}$ and can impact negatively on the sensorial perceptions.

\subsection{Grain/Cereal, Pseudocereal By-Product Utilisation in Bread and Effects on Sensory Profile}

Pre-fermentation as a pre-treatment processing of cereal ingredients such as the bran can be utilised to enhance the sensorial and nutritional profile of bread. The process allows for the release of aroma compounds. Pontonio et al. (2017) reported that bread formulated with fermented wheat bran and germ mixture recorded significantly higher in-vitro protein digestibility, protein scores, essential amino acid index and biological values compared to their unfermented and refined white wheat bread counterpart. The enriched bread was also characterized by a high content of dietary fibre and low glycaemic index determined in vivo [43]. The use of a dietary fibre preparation, derived from defatted rice bran, in bread revealed that breads with $5 \%$ and $10 \%$ rice bran fibre compared well to a control high-fibre bread available in the market in terms of the organoleptic attributes as evaluated by the participants [76]. The author suggested the defatting process possibly enhanced the functional properties of the rice bran fibre consequently resulting in bread with favourable sensory attributes. 
The incorporation of 'middling" fraction (M) of wholegrain (WM) and pearled (PM) barley in different ratios $(15 \%, 30 \%, 45 \%$, and $60 \%$ middlings with wheat flour) in bread and its effect on organoleptic perceptions has been reported. No significant difference was found in the acceptability of WM and PM breads. Up to a barley middlings (BM) inclusion level of 30\%, there were no significant differences in the acceptance of the breads. Above $30 \%$, however, the acceptability of the bread was shown to decrease significantly [78].

\subsection{Oilseed By-Product Utilisation in Bread and Effects on Sensory and Nutrition Profile}

Cakes from oilseeds including yellow linseed, sunflower seed, naked pumpkin seed, and walnut have been used in bread formulation at substitutions of $5 \%$ and $10 \%$ and their impact on bread sensory characteristics reported. Generally, enrichment of bread with cakes from the oil seeds was considered viable but in the case of pumpkin seed and sunflower seed cakes fortified flour blends, the use of hydrocolloid was recommended to improve visco-elastic properties [8]. Enrichment of wheat bread with either rootlets or fermented rootlets at the 5\% level resulted in bread that resonated with the organoleptic perceptions of panellists. In this study, the authors reported that, rootlet or fermented rootlet substituted bread above $10 \%$ had onerous organoleptic properties stemming from bitterness, acidulousness, and lack of sweetness [13]. Furthermore, the incorporation of either brewers spent grain or fermented brewers spent grain (using the lactic acid bacteria, L. plantarum FST 1.7) resulted in breads which were accepted from a sensory perspective up to a $10 \%$ level. The perceived sweetness of the bread was decreased upon increasing addition of brewers spent grain, and this effect was more pronounced upon addition of fermented brewers spent grain [14].

Bread enriched with nejayote solids $(3 \%, 6 \%, 9 \%)$ has been evaluated for its organoleptic perceptions by consumers. The authors reported that, the incorporation of the nejayote solids in the bread had no adverse effect on the textural perception by consumers. This was confirmed using a texture analyzer for objective texture analysis. There was no significant difference between the textural profile of the control and nejayote solid-supplemented breads in both the subjective and objective evaluation. The flavour and odour ranking of the nejayote-supplemented breads by consumers recorded lower scores as the amount of nejayote supplementation increased. The overall acceptability of the nejayote-supplemented bread (scores ranged from 7.14 to 7.47 ) and showed no difference to the "control" bread (7.47) [82].

\section{Bioavailability and Bioaccessibility of Bioactive Compounds from Functional Bread formulated with Plant-Based By-Products}

The effectiveness of functional bread to impact health effect relies on the bioavailability and bioaccessibility of the bioactive compounds following bread consumption. Bioavailability denotes the concentration of the ingested bioactive compound that reaches the systemic circulation with the potential of been utilised, whereas bioaccessibility refers to the concentration of bioactive compounds that potentially get absorbed from the lumen of the gut [98]. Van Dokkum et al. [99] reported that an increased excretion of minerals in the faeces usually occurs when coarse wheat bran-enriched bread is eaten, thus impacting on the bioavailability of minerals [99]. The physiological effect demonstrated by wheat bran and thus bran fibre when consumed in bran-enriched bread is suggested in part to be related to increasing faecal wet weight, defaecation frequency, and reduction of intestinal transit time [100]. Further research is required to better appreciate this mechanism. The bioactivity and health-effect of currant seed press residue, aleurone fraction from wheat bran, arabinoxylan from bran and rye bran, all of which are food by-products, is reported (Table 3). 
Table 3. Bioavailability/bioaccessibility, bioactive properties and health effects of bread formulated with plant-based by-products.

\begin{tabular}{|c|c|c|c|c|c|c|}
\hline Food by-Product & Type of Study & Sample Size & $\begin{array}{l}\text { Amount of Bioactive } \\
\text { Consumed }\end{array}$ & Findings & $\begin{array}{c}\text { Quantitative Changes } \\
\text { Induced by Food-by Product } \\
\text { Substitution }\end{array}$ & Reference \\
\hline Black currant seed press & $\begin{array}{l}\text { pre-post study } \\
\text { design }\end{array}$ & 36 (Healthy adults) & $\begin{array}{l}20 \mathrm{~g} \text { black currant } \\
\text { by-product } / \mathrm{d}\end{array}$ & $\begin{array}{l}\uparrow \text { serum } \alpha-, \gamma \text { - and } \downarrow \beta \text { - } \\
\text { tocopherol concentration. }\end{array}$ & $\begin{array}{c}\alpha \text { tocopherol } \uparrow(1.7 \%) \\
\beta \text { tocopherol } \downarrow(5.3 \%) \\
\gamma \text { tocopherol } \uparrow(20.8 \%) \text { (change } \\
\text { from baseline) }\end{array}$ & [21] \\
\hline $\begin{array}{l}\text { Aleurone fraction of wheat } \\
\text { bran }\end{array}$ & $\begin{array}{l}\text { single-blind, } \\
\text { randomised, } \\
\text { cross-over study }\end{array}$ & $\begin{array}{l}15 \text { (urine sampling } \\
\text { periods) and } 5 \text { (blood } \\
\text { sampling periods) } \\
\text { (Healthy adults) }\end{array}$ & $\begin{array}{c}\text { approx. } 94(\mathrm{AB}-94) \text { and } \\
190 \mathrm{~g}(\mathrm{AB}-190) \\
\text { aleurone-rich breads } \\
\text { containing approx. } 43 \\
\text { and } 87 \text { mg of total ferulic } \\
\text { acid respectively }\end{array}$ & $\uparrow$ bioavailability of ferulic acid & $\begin{array}{l}\% \text { bioavailability-control-4\%, } \\
\text { AB-94- } 8 \% \text { and AB-190 5\% }\end{array}$ & [101] \\
\hline $\begin{array}{l}\text { Arabinoxylan-oligosaccharides } \\
\text { from rye bran treated with } \\
\text { thermophilic endoxylanase }\end{array}$ & $\begin{array}{l}\text { randomised, } \\
\text { double-blind, } \\
\text { placebo controlled } \\
\text { cross-over study }\end{array}$ & 40 (Healthy adults) & $2.20 \mathrm{~g}$ arabinoxylan & $\begin{array}{l}\uparrow \text { faecal butyrate and acetate } \\
\text { and } \downarrow \text { propionate concentration }\end{array}$ & $\begin{array}{c}\text { butyrate } \uparrow(29.4 \%) \text {, acetate } \\
\uparrow(17.2 \%) \text {, propionate } \downarrow(1.7 \%) \\
\text { and total acetate, propionate } \\
\text { and butyrate } \uparrow(15.7 \%) \\
\text { (changes from baseline) }\end{array}$ & [25] \\
\hline $\begin{array}{l}\text { In situ-produced arabinoxylan } \\
\text { from rye bran }\end{array}$ & $\begin{array}{l}\text { randomised, } \\
\text { double-blind, } \\
\text { controlled, } \\
\text { cross-over study }\end{array}$ & 27 (Healthy adults) & $1.90 \%$ of dry matter & $\begin{array}{c}\uparrow \text { faecal total short chain fatty } \\
\text { acids concentration } \\
\text { particularly butyric acid }\end{array}$ & $\begin{array}{c}\text { acetic acid } \uparrow(27.9 \%) \text {, propionic } \\
\text { acid } \uparrow(28.8 \%), \text { butyric acid } \\
\uparrow(41.5 \%) \text { and total short chain } \\
\text { fatty acids } \uparrow(35.7 \%)\end{array}$ & [26] \\
\hline Arabinoxylan fibre (AX) & $\begin{array}{l}\text { randomised, } \\
\text { crossover design }\end{array}$ & 14 (Healthy adults) & 6 and $12 \mathrm{~g} \mathrm{AX}$ fibre & $\begin{array}{l}\downarrow \text { peak postprandial glucose } \\
\text { concentration }\end{array}$ & $\begin{array}{c}\text { incremental area under the } \\
\text { glucose curve: } 6 \mathrm{~g} \mathrm{AX}- \\
\downarrow(\sim 23.8 \%) \text { and } 12 \mathrm{~g} \text { AX- } \\
\downarrow(\sim 42.9 \%) \text { and incremental } \\
\text { area under insulin curve: } 6 \mathrm{~g} \\
\text { AX- } \downarrow(\sim 19.6 \%) \text { and } 12 \mathrm{~g} \mathrm{AX} \\
\downarrow(\sim 42.9 \%)\end{array}$ & [102] \\
\hline $\begin{array}{l}\text { Wheat bran extract rich in } \\
\text { arabinoxylan oligosaccharides } \\
\text { (AXOS) }\end{array}$ & $\begin{array}{l}\text { randomised } \\
\text { cross-over study }\end{array}$ & 19 (Healthy adults) & 18.4 AXOS g/portion & $\begin{array}{c}\downarrow \text { glycaemic response. } \uparrow \text { acetate, } \\
\text { propionate, butyrate and total } \\
\text { short chain fatty acid } \\
\text { concentration in the morning. }\end{array}$ & $\begin{array}{l}\text { change in glucose incremental } \\
\text { area under the curve }(0-120 \\
\text { min) } \downarrow(15.8 \%) \text { and } \downarrow(18.5 \%) \text { for } \\
\text { (0-180 min) when compared to } \\
\text { control bread }\end{array}$ & [20] \\
\hline $\begin{array}{l}\text { Concentrated arabinoxylan } \\
\text { from wheat }\end{array}$ & $\begin{array}{l}\text { acute, randomised } \\
\text { cross-over } \\
\text { intervention study }\end{array}$ & $\begin{array}{c}15 \text { (Adults with } \\
\text { Metabolic syndrome) }\end{array}$ & $7 \mathrm{~g}$ of arabinoxylan & $\uparrow$ satiety sensation & $\begin{array}{c}\text { incremental area under the } \\
\text { curve for satiety change } \\
\uparrow(26 \%) \text {, }\end{array}$ & [23] \\
\hline
\end{tabular}


Table 3. Cont.

\begin{tabular}{|c|c|c|c|c|c|c|}
\hline Food by-Product & Type of Study & Sample Size & $\begin{array}{l}\text { Amount of Bioactive } \\
\text { Consumed }\end{array}$ & Findings & $\begin{array}{c}\text { Quantitative Changes } \\
\text { Induced by Food-by Product } \\
\text { Substitution }\end{array}$ & Reference \\
\hline Wheat bran and aleurone & $\begin{array}{l}\text { randomized } \\
\text { controlled, } \\
\text { cross-over }\end{array}$ & 14 (Healthy adults) & $50 \mathrm{~g}$ each & $\begin{array}{l}\uparrow \text { postprandial plasma betaine } \\
\text { concentrations with } \\
\text { consumption of minimally } \\
\text { processed wheat bran }\end{array}$ & $\begin{array}{l}\text { postprandial betaine } \\
\text { concentration } \uparrow(49 \%)\end{array}$ & [19] \\
\hline Rye bran & $\begin{array}{l}\text { randomized } \\
\text { crossover }\end{array}$ & 12 (Healthy adults) & $\begin{array}{c}\text { In a flour basis of } 65 \% \\
\text { white wheat flour and } \\
35 \% \text { bran, whereas the } \\
\text { commercial sourdough } \\
\text { rye bread contained } 16 \% \\
\text { bran }\end{array}$ & $\begin{array}{l}\uparrow \text { sulfonated phenylacetamides } \\
\text { compounds in blood plasma } \\
\text { specifically } \\
\text { hydroxy-N-(2-hydroxyphenyl) } \\
\text { acetamide and } \\
\text { N-(2-hydroxyphenyl) } \\
\text { acetamide }\end{array}$ & N/A & [103] \\
\hline Rye bran & $\begin{array}{l}\text { randomised } \\
\text { crossover trial }\end{array}$ & 20 (Healthy adults) & $\begin{array}{l}\text { The portions of rye } \\
\text { breads weighed } \\
24.1-28.1 \mathrm{~g} \text { and those of } \\
\text { wheat breads, 20.8-25.0 } \\
\text { g. A minimum of } 4-5 \\
\text { portions of the test } \\
\text { breads had to be eaten } \\
\text { each day. }\end{array}$ & insulin secretion & $\begin{array}{l}\text { plasma glucose } \uparrow(0.7 \%) \text { and } \\
\text { plasma insulin } \downarrow(6 \%)-\end{array}$ & [104] \\
\hline
\end{tabular}

NB: Adults participants implies participants were aged 18 years and over. N/A—Not applicable. 
Helbig et al. [21], in a four-week period, studied the effect of consuming bread enriched with black currant seed cake on the concentration of tocopherol in serum and stool of 36 women. The authors reported that, the consumption of the press residue enriched-bread led to significant increase in serum total $\beta-, \gamma-\delta$ - and tocopherol concentration [21] possibly from the residual oils in the seed cake. The tocopherols particularly $\gamma-\delta$ - and tocopherol demonstrate potent anticancer properties possibly due to their ability to demonstrate antioxidant properties scavenging reactive nitrogen species in addition to reactive oxygen species [105].

The bran of wheat obtained from the processing of whole-wheat kernel contains $45-50 \%$ of the aleurone layer. These aleurone fractions of bran have been used in bread formulation. Bresciani et al. [101] reported that, the intake of low concentration of aleurone-enriched bread contributes equal concentration of ferulic acid in the plasma as wholegrain bread. The authors detected phenolic metabolites in urine (12) and plasma (4) respectively. The metabolites detected included high concentrations of ferulic acid-4'-O-sulfate, dihydroferulic acid-4'-O-sulfate, dihydroferulic acid-O-glucuronide and sulfate and glucuronic acid conjugates of phenolic acids. A significant increase in the bioavailability of ferulic acid was reported following the consumption of the aleurone-enriched bread. The authors postulated that, absorbed phenolic metabolites following the consumption of wholegrain product impact health benefits, the consumption of lower doses of refined products enriched with wheat bran aleurone fraction could impact similar benefits [101].

The bran of most grains (including wheat and rye) contain high concentration of arabinoxylan. The formulation of bread with different treated forms of arabinoxylan, including in situ-produced arabinoxylan from rye bran [26], arabinoxylan-oligosaccharides treated with thermophilic endoxylanase [25], extracted arabinoxylan-rich fibre from processed wheat flour [102], and arabinoxylan oligosaccharides-rich wheat bran extract [20], have been evaluated for the health outcomes associated with their consumption. Health outcomes reported include blunting post-prandial glucose response [102], attenuating insulin response [102], increased faecal butyrate, bifidobacteria and lactobacilli concentration, reduced iso-valerate and fatty acids attributed to the protein fermentation [25], and favourable modulation of intestinal fermentation and overall gastrointestinal properties in healthy humans [26] with enhanced breath $\mathrm{H}_{2}$ concentration and circulating SCFA [20]. Additionally, bread enriched with concentrated arabinoxylan significantly improved satiety sensations [23].

In another study that was carried out in two stages, post-prandial plasma betaine and other methyl donor-related responses following the consumption of minimally processed wheat bran or wheat aleurone, or wheat aleurone added to bread was reported. Participants consumed two different bread types (enriched with minimally processed wheat bran and minimally processed wheat aleurone) and a control white bread in the first study. In the second study, the participants consumed one treatment bread (wheat aleurone incorporated into bread) and a "control" white bread. The authors reported that, plasma betaine concentrations increased significantly from $30 \mathrm{~min}$ to $3 \mathrm{~h}$ following the consumption of both bran and aleurone-enriched breads compared with the control; however, the aleurone-enriched bread produced significantly higher responses than bran counterpart in the first study. For the second study, plasma betaine concentrations were significantly and substantially higher following the consumption of the aleurone-enriched bread compared with the control bread. Again small, but significant, increase was observed in dimethylglycine and folate measures in response to consumption of the aleurone- enriched bread. Peak plasma betaine concentrations, which were 1.70-1.80 times the baseline concentration, were achieved earlier following the consumption of the minimally processed aleurone compared with the aleurone-enriched bread [19]. The consumption of a diet with a higher concentration of betaine, an amino acid, is associated with lower insulin resistance [106].

Hanhineva et al. [103] investigated whether variations in the profile of bioactive compounds in blood plasma following the consumption of three breads containing rye bran $(100 \%$ whole-grain sourdough rye bread or white wheat bread enriched with native unprocessed rye bran or bioprocessed rye bran) when compared with a control plain white wheat bread existed in 12 healthy middle-aged men and women. Two sulfonated phenylacetamides, hydroxy-N-(2-hydroxyphenyl) acetamide and 
$\mathrm{N}-(2$-hydroxyphenyl) acetamide, potentially derived from the benzoxazinoid metabolites, were among the most discriminant postprandial plasma biomarkers distinguishing the intake of breads containing whole-meal rye or rye bran from the control white wheat bread. Benzoxazinoids have been reported to militate against the proliferation of prostate cancer cell lines [107]. In another study that investigated the glycaemic response of the long-term intake of rye bran-enriched bread in hypercholesterolemic post-menopausal women, the authors observed that the consumption of fibre-rich rye bread did not alter insulin, but rather enhanced insulin secretion, possibly improving the activity of $\beta$-cell function [104].

In summary, the review of literature highlights potential health effect associated with the consumption of bread enriched with plant-based food by-products including attenuating glucose and insulin release, improved satiety sensations and increased short chain fatty acids production. However, more studies are warranted to establish the potential health effects associated with the long term consumption of the breads enriched with plant-based food by-products as most of the studies conducted were acute in design.

\section{Conclusions}

Food by-products, including rice bran, wheat bran, rootlets, brewers spent grain, oat bran, grape pomace, and seed extract, contain high concentrations of bioactive compounds, including polyphenols, carotenoids, tocochromanols, arabinoxylan, beta-glucans, essential fatty acids, and gamma amino butyric acid, that have the potential to demonstrate health effects. The increased bioactivity of functional bread has been observed following the incorporation of flour from the plant-based food by-products. In most cases, bread formulated with up to $6 \%$ flour from food by-products had enhanced qualities. In terms of sensory acceptability of bread, formulations up to an average of $5 \%$ resulted in bread with appealing organoleptic perceptions to consumers. The application of pre-treatment of the plant-based food by-products using fermentation and thermal processes impacted on the properties of the by-products consequently giving loaves with enhanced qualities. The utilisation of the plant-based by-products in bread demonstrated favourable bioactive properties in human blood with enhanced bioavailability and bioaccessibility of the bioactive ingredients. Further research must explore the use of clinical trials to validate the health effect associated with the long-term consumption of bread enriched with plant-based by-products. Additionally, new research areas should investigate the bioactivity of other underutilised plant-based food by-products, their possible incorporation in bread formulation, bioactivity in bread, and effect on physical, sensory, and nutritional properties.

Author Contributions: All authors contributed towards the writing, revision, and production of the final draft of the manuscript. All authors have read and agreed to the published version of the manuscript.

Funding: This research received no external funding and The APC was funded by Elaine Rush.

Acknowledgments: The authors are grateful to the Riddet Institute, a New Zealand Centre of Research Excellence, funded by the Tertiary Education Commission, for awarding Isaac Amoah with the scholarship.

Conflicts of Interest: The authors declare no conflict of interest.

\section{References}

1. Onipe, O.O.; Jideani, A.I.O.; Beswa, D. Composition and functionality of wheat bran and its application in some cereal food products. Int. J. Food Sci. Technol. 2015, 50, 2509-2518. [CrossRef]

2. Sporin, M.; Avbelj, M.; Kovac, B.; Mozina, S.S. Quality characteristics of wheat flour dough and bread containing grape pomace flour. Food Sci. Technol. Int. 2018, 24, 251-263. [CrossRef]

3. Bordiga, M.; Travaglia, F.; Locatelli, M.; Arlorio, M.; Coïsson, J.D. Spent grape pomace as a still potential by-product. Int. J. Food Sci. Technol. 2015, 50, 2022-2031. [CrossRef]

4. Chareonthaikij, P.; Uan-On, T.; Prinyawiwatkul, W. Effects of pineapple pomace fibre on physicochemical properties of composite flour and dough, and consumer acceptance of fibre-enriched wheat bread. Int. J. Food Sci. Technol. 2016, 51, 1120-1129. [CrossRef] 
5. Banerjee, S.; Ranganathan, V.; Patti, A.; Arora, A. Valorisation of pineapple wastes for food and therapeutic applications. Trends Food Sci. Technol. 2018, 82, 60-70. [CrossRef]

6. Lafarga, T.; Gallagher, E.; Bademunt, A.; Viñas, I.; Bobo, G.; Villaró, S.; Aguiló-Aguayo, I. Bioaccessibility, physicochemical, sensorial, and nutritional characteristics of bread containing broccoli co-products. J. Food Process. Preserv. 2019, 43, e13861. [CrossRef]

7. Wirkijowska, A.; Zarzycki, P.; Sobota, A.; Nawrocka, A.; Blicharz-Kania, A.; Andrejko, D. The possibility of using by-products from the flaxseed industry for functional bread production. LWT 2020, 118, 108860. [CrossRef]

8. Tarek-Tilistyák, J.; Agócs, J.; Lukács, M.; Dobró-Tóth, M.; Juhász-Román, M.; Dinya, Z.; Jekő, J.; Máthé, E. Novel breads fortified through oilseed and nut cakes. Acta Aliment. 2014, 43, 444-451. [CrossRef]

9. Wadhwa, M.; Bakshi, M.P.S. Utilization of Fruit and Vegetable Wastes as Livestock Feed and as Substrates for Generation of Other Value-Added Products; FAO: Rome, Italy, 2013.

10. Papargyropoulou, E.; Lozano, R.; Steinberger, J.K.; Wright, N.; bin Ujang, Z. The food waste hierarchy as a framework for the management of food surplus and food waste. J. Clean. Prod. 2014, 76, 106-115. [CrossRef]

11. Sohail, M.; Rakha, A.; Butt, M.S.; Iqbal, M.J.; Rashid, S. Rice bran nutraceutics: A comprehensive review. Crit. Rev. Food Sci. Nutr. 2017, 57, 3771-3780. [CrossRef]

12. Brenes, A.; Viveros, A.; Chamorro, S.; Arija, I. Use of polyphenol-rich grape by-products in monogastric nutrition. A review. Anim. Feed Sci. Technol. 2016, 211, 1-17. [CrossRef]

13. Waters, D.M.; Kingston, W.; Jacob, F.; Titze, J.; Arendt, E.K.; Zannini, E. Wheat bread biofortification with rootlets, a malting by-product. J. Sci. Food Agric. 2013, 93, 2372-2383. [CrossRef] [PubMed]

14. Waters, D.M.; Jacob, F.; Titze, J.; Arendt, E.K.; Zannini, E. Fibre, protein and mineral fortification of wheat bread through milled and fermented brewer's spent grain enrichment. Eur. Food Res. Technol. 2012, 235, 767-778. [CrossRef]

15. Shyu, Y.S.; Lu, T.C.; Lin, C.C. Functional analysis of unfermented and fermented citrus peels and physical properties of citrus peel-added doughs for bread making. J. Food Sci. Technol. 2014, 51, 3803-3811. [CrossRef] [PubMed]

16. Frutos, M.J.; Guilabert-Antón, L.; Tomás-Bellido, A.; Hernández-Herrero, J.A. Effect of artichoke (Cynara scolymus L.) fiber on textural and sensory qualities of wheat bread. Food Sci. Technol. Int. 2008, 14, 49-55. [CrossRef]

17. Nour, V.; Ionica, M.E.; Trandafir, I. Bread enriched in lycopene and other bioactive compounds by addition of dry tomato waste. J. Food Sci. Technol. 2015, 52, 8260-8267. [CrossRef]

18. Kourkouta, L.; Koukourikos, K.; Iliadis, C.; Ouzounakis, P.; Monios, A.; Tsaloglidou, A. Bread and health. J. Pharm. Pharmacol. 2017, 5, 821-826. [CrossRef]

19. Keaveney, E.M.; Price, R.K.; Hamill, L.L.; Wallace, J.M.; McNulty, H.; Ward, M.; Strain, J.J.; Ueland, P.M.; Molloy, A.M.; Piironen, V.; et al. Postprandial plasma betaine and other methyl donor-related responses after consumption of minimally processed wheat bran or wheat aleurone, or wheat aleurone incorporated into bread. Br. J. Nutr. 2015, 113, 445-453. [CrossRef]

20. Boll, E.V.; Ekstrom, L.M.; Courtin, C.M.; Delcour, J.A.; Nilsson, A.C.; Bjorck, I.M.; Ostman, E.M. Effects of wheat bran extract rich in arabinoxylan oligosaccharides and resistant starch on overnight glucose tolerance and markers of gut fermentation in healthy young adults. Eur. J. Nutr. 2016, 55, 1661-1670. [CrossRef]

21. Helbig, D.; Wagner, A.; Schubert, R.; Jahreis, G. Tocopherol isomer pattern in serum and stool of human following consumption of black currant seed press residue administered in whole grain bread. Clin. Nutr. 2009, 28, 662-667. [CrossRef]

22. Birch, C.S.; Bonwick, G.A. Ensuring the future of functional foods. Int. J. Food Sci. Technol. 2018, 54, 1467-1485. [CrossRef]

23. Hartvigsen, M.L.; Gregersen, S.; Laerke, H.N.; Holst, J.J.; Bach Knudsen, K.E.; Hermansen, K. Effects of concentrated arabinoxylan and beta-glucan compared with refined wheat and whole grain rye on glucose and appetite in subjects with the metabolic syndrome: A randomized study. Eur. J. Clin. Nutr. 2014, 68, 84-90. [CrossRef] [PubMed]

24. Kikuchi, Y.; Nozaki, S.; Makita, M.; Yokozuka, S.; Fukudome, S.I.; Yanagisawa, T.; Aoe, S. Effects of whole grain wheat bread on visceral fat obesity in Japanese subjects: A randomized double-blind study. Plant Foods Hum. Nutr. 2018, 73, 1-5. [CrossRef] [PubMed] 
25. Walton, G.E.; Lu, C.I.; Trogh, I.; Arnaut, F.; Gibson, G.R. A randomised, double-blind, placebo controlled cross-over study to determine the gastrointestinal effects of consumption of arabinoxylan-oligosaccharides enriched bread in healthy volunteers. Nutr. J. 2012, 11, 36. [CrossRef]

26. Damen, B.; Cloetens, L.; Broekaert, W.F.; Francois, I.; Lescroart, O.; Trogh, I.; Arnaut, F.; Welling, G.W.; Wijffels, J.; Delcour, J.A.; et al. Consumption of breads containing in situ-produced arabinoxylan oligosaccharides alters gastrointestinal effects in healthy volunteers. J. Nutr. 2012, 142, 470-477. [CrossRef]

27. Martins, Z.E.; Pinho, O.; Ferreira, I.M.P.L.V.O. Food industry by-products used as functional ingredients of bakery products. Trends Food Sci. Technol. 2017, 67, 106-128. [CrossRef]

28. Gomez, M.; Martinez, M.M. Fruit and vegetable by-products as novel ingredients to improve the nutritional quality of baked goods. Crit. Rev. Food Sci. Nutr. 2018, 58, 2119-2135. [CrossRef]

29. Kowalska, H.; Czajkowska, K.; Cichowska, J.; Lenart, A. What's new in biopotential of fruit and vegetable by-products applied in the food processing industry. Trends Food Sci. Technol. 2017, 67, 150-159. [CrossRef]

30. De Camargo, A.C.; Schwember, A.R.; Parada, R.; Garcia, S.; Maróstica Júnior, M.R.; Franchin, M.; Regitano-d'Arce, M.A.B.; Shahidi, F. Opinion on the hurdles and potential health benefits in value-added use of plant food processing by-products as sources of phenolic compounds. Int. J. Mol. Sci. 2018, 19, 3498. [CrossRef]

31. Shahidi, F.; Yeo, J.D. Insoluble-Bound Phenolics in Food. Molecules 2016, 21, 1216. [CrossRef]

32. Champ, C.E.; Kundu-Champ, A. Maximizing polyphenol content to uncork the relationship between wine and cancer. Front. Nutr. 2019, 6, 44. [CrossRef] [PubMed]

33. Cao, H.; Ou, J.; Chen, L.; Zhang, Y.; Szkudelski, T.; Delmas, D.; Daglia, M.; Xiao, J. Dietary polyphenols and type 2 diabetes: Human study and clinical trials. Crit. Rev. Food Sci. Nutr. 2018, 59, 1-19. [CrossRef]

34. Bajerska, J.; Mildner-Szkudlarz, S.; Jeszka, J.; Szwengiel, A. Catechin stability, antioxidant properties and sensory profiles of rye breads fortified with green tea extracts. J. Food Nutr. Res. 2010, 49, 104-111.

35. Rauf, A.; Imran, M.; Orhan, I.E.; Bawazeer, S. Health perspectives of a bioactive compound curcumin: A review. Trends Food Sci. Technol. 2018, 74, 33-45. [CrossRef]

36. Lesinski, G.B.; Reville, P.K.; Mace, T.A.; Young, G.S.; Ahn-Jarvis, J.; Thomas-Ahner, J.; Vodovotz, Y.; Ameen, Z.; Grainger, E.; Riedl, K.; et al. Consumption of soy isoflavone enriched bread in men with prostate cancer is associated with reduced proinflammatory cytokines and immunosuppressive cells. Cancer Prev. Res. 2015, 8, 1036-1044. [CrossRef]

37. Sharif, M.K.; Butt, M.S.; Anjum, F.M.; Khan, S.H. Rice bran: A novel functional ingredient. Crit. Rev. Food Sci. Nutr. 2014, 54, 807-816. [CrossRef]

38. Liu, Y.Q.; Strappe, P.; Shang, W.T.; Zhou, Z.K. Functional peptides derived from rice bran proteins. Crit. Rev. Food Sci. Nutr. 2017, 59, 1-8. [CrossRef]

39. Pang, Y.; Ahmed, S.; Xu, Y.; Beta, T.; Zhu, Z.; Shao, Y.; Bao, J. Bound phenolic compounds and antioxidant properties of whole grain and bran of white, red and black rice. Food Chem. 2018, 240, 212-221. [CrossRef]

40. Martin, M.; Deussen, A. Effects of natural peptides from food proteins on angiotensin converting enzyme activity and hypertension. Crit. Rev. Food Sci. Nutr. 2017, 59, 1-20. [CrossRef]

41. Li-Chan, E.C.Y. Bioactive peptides and protein hydrolysates: Research trends and challenges for application as nutraceuticals and functional food ingredients. Curr. Opin. Food Sci. 2015, 1, 28-37. [CrossRef]

42. Xie, X.S.; Cui, S.W.; Li, W.; Tsao, R. Isolation and characterization of wheat bran starch. Food Res. Int. 2008, 41, 882-887. [CrossRef]

43. Pontonio, E.; Lorusso, A.; Gobbetti, M.; Rizzello, C.G. Use of fermented milling by-products as functional ingredient to develop a low-glycaemic index bread. J. Cereal Sci. 2017, 77, 235-242. [CrossRef]

44. Coda, R.; Karki, I.; Nordlund, E.; Heinio, R.L.; Poutanen, K.; Katina, K. Influence of particle size on bioprocess induced changes on technological functionality of wheat bran. Food Microbiol. 2014, 37, 69-77. [CrossRef] [PubMed]

45. Smuda, S.S.; Mohsen, S.M.; Olsen, K.; Aly, M.H. Bioactive compounds and antioxidant activities of some cereal milling by-products. J. Food Sci. Technol. 2018, 55, 1134-1142. [CrossRef]

46. Giordano, D.; Locatelli, M.; Travaglia, F.; Bordiga, M.; Reyneri, A.; Coisson, J.D.; Blandino, M. Bioactive compound and antioxidant activity distribution in roller-milled and pearled fractions of conventional and pigmented wheat varieties. Food Chem. 2017, 233, 483-491. [CrossRef]

47. Balandrán-Quintana, R.R.; Mercado-Ruiz, J.N.; Mendoza-Wilson, A.M. Wheat Bran Proteins: A Review of Their Uses and Potential. Food Rev. Int. 2015, 31, 279-293. [CrossRef] 
48. Górnaś, P.; Radenkovs, V.; Pugajeva, I.; Soliven, A.; Needs, P.W.; Kroon, P.A. Varied Composition of Tocochromanols in Different Types of Bran: Rye, Wheat, Oat, Spelt, Buckwheat, Corn, and Rice. Int. J. Food Prop. 2016, 19, 1757-1764. [CrossRef]

49. Gornas, P.; Misina, I.; Gravite, I.; Soliven, A.; Kaufmane, E.; Seglina, D. Tocochromanols composition in kernels recovered from different apricot varieties: RP-HPLC/FLD and RP-UPLC-ESI/MS(n) study. Nat. Prod. Res. 2015, 29, 1222-1227. [CrossRef]

50. Mohd Mutalip, S.S.; Ab-Rahim, S.; Rajikin, M.H. Vitamin E as an Antioxidant in Female Reproductive Health. Antioxidants 2018, 7, 22. [CrossRef]

51. Rose, D.J.; Inglett, G.E.; Liu, S.X. Utilisation of corn (Zea mays) bran and corn fiber in the production of food components. J. Sci. Food Agric. 2010, 90, 915-924. [CrossRef]

52. Blackwood, A.D.; Salter, J.; Dettmar, P.W.; Chaplin, M.F. Dietary fibre, physicochemical properties and their relationship to health. J. R. Soc. Promot. Health 2000, 120, 242-247. [CrossRef] [PubMed]

53. Sajdakowska, M.; Gebski, J.; Zakowska-Biemans, S.; Jezewska-Zychowicz, M. Willingness to eat bread with health benefits: Habits, taste and health in bread choice. Public Health 2019, 167, 78-87. [CrossRef] [PubMed]

54. Ham, H.; Woo, K.S.; Park, J.Y.; Lee, B.; Choi, H.S.; Choi, Y.H.; Lee, J.; Lee, Y.Y. Antioxidant compounds and antioxidant activities of methanolic extracts from milling fractions of oat. Korean J. Food Nutr. 2016, 45, 1681-1684. [CrossRef]

55. Khan, M.A.; Nadeem, M.; Rakha, A.; Shakoor, S.; Shehzad, A.; Khan, M.R. Structural Characterization of Oat Bran $(1 \rightarrow 3)$, (1 $\rightarrow 4)$ - $\beta$-D-Glucans by Lichenase Hydrolysis Through High-Performance Anion Exchange Chromatography with Pulsed Amperometric Detection. Int. J. Food Prop. 2015, 19, 929-935. [CrossRef]

56. Maltini, E.; Torreggiani, D.; Venir, E.; Bertolo, G. Water activity and the preservation of plant foods. Food Chem. 2003, 82, 79-86. [CrossRef]

57. Larrauri, J.A.; Rupérez, P.; Saura-Calixto, F. Mango peel fibres with antioxidant activity. Z. Für Lebensm. Und-Forsch. A 1997, 205, 39-42. [CrossRef]

58. Jiang, L.N.; Liu, Y.B.; Li, B.H. Lycopene exerts anti-inflammatory effect to inhibit prostate cancer progression. Asian J. Androl. 2018, 21, 80-85. [CrossRef]

59. Anang, D.A.; Pobee, R.A.; Antwi, E.; Obeng, E.M.; Atter, A.; Ayittey, F.K.; Boateng, J.T. Nutritional, microbial and sensory attributes of bread fortified with defatted watermelon seed flour. Int. J. Food Sci. Technol. 2018, 53, 1468-1475. [CrossRef]

60. Kristensen, M.; Damgaard, T.W.; Sorensen, A.D.; Raben, A.; Lindelov, T.S.; Thomsen, A.D.; Bjergegaard, C.; Sorensen, H.; Astrup, A.; Tetens, I. Whole flaxseeds but not sunflower seeds in rye bread reduce apparent digestibility of fat in healthy volunteers. Eur. J. Clin. Nutr. 2008, 62, 961-967. [CrossRef]

61. Ribas-Agusti, A.; Martin-Belloso, O.; Soliva-Fortuny, R.; Elez-Martinez, P. Food processing strategies to enhance phenolic compounds bioaccessibility and bioavailability in plant-based foods. Crit. Rev. Food Sci. Nutr. 2017, 58, 1-18. [CrossRef]

62. Skenderidis, P.; Mitsagga, C.; Giavasis, I.; Petrotos, K.; Lampakis, D.; Leontopoulos, S.; Hadjichristodoulou, C.; Tsakalof, A. The in vitro antimicrobial activity assessment of ultrasound assisted Lycium barbarum fruit extracts and pomegranate fruit peels. J. Food Meas. Charact. 2019, 13, 2017-2031. [CrossRef]

63. Colantuono, A.; Ferracane, R.; Vitaglione, P. Potential bioaccessibility and functionality of polyphenols and cynaropicrin from breads enriched with artichoke stem. Food Chem. 2018, 245, 838-844. [CrossRef] [PubMed]

64. Longoria-García, S.; Cruz-Hernández, M.A.; Flores-Verástegui, M.I.M.; Contreras-Esquivel, J.C.; Montañez-Sáenz, J.C.; Belmares-Cerda, R.E. Potential functional bakery products as delivery systems for prebiotics and probiotics health enhancers. J. Food Sci. Technol. 2018, 55, 833-845. [CrossRef] [PubMed]

65. Ananingsih, V.K.; Gao, J.; Zhou, W. Impact of green tea extract and fungal alpha-amylase on dough proofing and steaming. Food Bioproc. Technol. 2013, 6, 3400-3411. [CrossRef]

66. Pathak, D.; Majumdar, J.; Raychaudhuri, U.; Chakraborty, R. Study on enrichment of whole wheat bread quality with the incorporation of tropical fruit by-product. Int. Food Res. J. 2017, 24, 238-246.

67. Chang, R.C.; Li, C.Y.; Shiau, S.Y. Physico-chemical and sensory properties of bread enriched with lemon pomace fiber. Czech J. Food Sci. 2015, 33, 180-185. [CrossRef]

68. Hoye, C., Jr.; Ross, C.F. Total phenolic content, consumer acceptance, and instrumental analysis of bread made with grape seed flour. J. Food Sci. 2011, 76, S428-S436. [CrossRef] [PubMed]

69. Peng, X.; Ma, J.; Cheng, K.; Jiang, Y.; Chen, F.; Wang, M. The effects of grape seed extract fortification on the antioxidant activity and quality attributes of bread. Food Chem. 2010, 119, 49-53. [CrossRef] 
70. Gül, H.; Şen, H. Effects of pomegranate seed flour on dough rheology and bread quality. CYTA J. Food 2017, 15, 622-628. [CrossRef]

71. Sun-Waterhouse, D.; Sivam, A.S.; Cooney, J.; Zhou, J.; Perera, C.O.; Waterhouse, G.I.N. Effects of added fruit polyphenols and pectin on the properties of finished breads revealed by HPLC/LC-MS and size-exclusion HPLC. Food Res. Int. 2011, 44, 3047-3056. [CrossRef]

72. Sivam, A.S.; Sun-Waterhouse, D.; Waterhouse, G.I.N.; Quek, S.Y.; Perera, C.O. Physicochemical properties of bread dough and finished bread with added pectin fiber and phenolic antioxidants. J. Food Sci. 2011, 76, 3. [CrossRef] [PubMed]

73. Martins, Z.E.; Pinho, O.; Ferreira, I.M.P.L.V.O.; Jekle, M.; Becker, T. Development of fibre-enriched wheat breads: Impact of recovered agroindustrial by-products on physicochemical properties of dough and bread characteristics. Eur. Food Res. Technol. 2017, 243, 1973-1988. [CrossRef]

74. Bchir, B.; Rabetafika, H.N.; Paquot, M.; Blecker, C. Effect of pear, apple and date fibres from cooked fruit by-products on dough performance and bread quality. Food Bioproc. Technol. 2014, 7, 1114-1127. [CrossRef]

75. Lima, I.; Guraya, H.; Champagne, E. The functional effectiveness of reprocessed rice bran as an ingredient in bakery products. Nahrung/Food 2002, 46, 112-117. [CrossRef]

76. Abdul-Hamid, A.; Luan, Y.S. Functional properties of dietary fibre prepared from defatted rice bran. Food Chem. 2000, 68, 15-19. [CrossRef]

77. Farahmand, E.; Razavi, S.; Yarmand, M.; Morovatpour, M. Development of Iranian rice-bran sourdough breads: Physicochemical, microbiological and sensorial characterisation during the storage period. Qual. Assur. Saf. Crop. Foods 2015, 7, 295-303. [CrossRef]

78. Sullivan, P.; O'Flaherty, J.; Brunton, N.; Arendt, E.; Gallagher, E. The utilisation of barley middlings to add value and health benefits to white breads. J. Food Eng. 2011, 105, 493-502. [CrossRef]

79. Banu, I.; Măcelaru, I.; Aprodu, I. Bioprocessing for improving the rheological properties of dough and quality of the wheat bread supplemented with oat bran. J. Food Process. Preserv. 2017, 41, e13112. [CrossRef]

80. Hemdane, S.; Langenaeken, N.A.; Jacobs, P.J.; Verspreet, J.; Delcour, J.A.; Courtin, C.M. Study of the intrinsic properties of wheat bran and pearlings obtained by sequential debranning and their role in bran-enriched bread making. J. Cereal Sci. 2016, 71, 78-85. [CrossRef]

81. Atalay, M.H.; Bilgicli, N.; Elgün, A.; Demir, M.K. Effects of buckwheat (Fagopyrum esculentummoench) milling products, transglutaminase and sodium stearoyl-2-lactylate on bread properties. J. Food Process. Preserv. 2013, 37, 1-9. [CrossRef]

82. Acosta-Estrada, B.A.; Lazo-Vélez, M.A.; Nava-Valdez, Y.; Gutiérrez-Uribe, J.A.; Serna-Saldívar, S.O. Improvement of dietary fiber, ferulic acid and calcium contents in pan bread enriched with nejayote food additive from white maize (Zea mays). J. Cereal Sci. 2014, 60, 264-269. [CrossRef]

83. Pojić, M.; Dapčević Hadnađev, T.; Hadnađev, M.; Rakita, S.; Brlek, T. Bread supplementation with hemp seed cake: A by-product of hemp oil processing. J. Food Qual. 2015, 38, 431-440. [CrossRef]

84. Salgado, J.M.; Rodrigues, B.S.; Donado-Pestana, C.M.; dos Santos Dias, C.T.; Morzelle, M.C. Cupuassu (Theobroma grandiflorum) peel as potential source of dietary fiber and phytochemicals in whole-bread preparations. Plant Foods Hum. Nutr. 2011, 66, 384-390. [CrossRef] [PubMed]

85. Elleuch, M.; Bedigian, D.; Roiseux, O.; Besbes, S.; Blecker, C.; Attia, H. Dietary fibre and fibre-rich by-products of food processing: Characterisation, technological functionality and commercial applications: A review. Food Chem. 2011, 124, 411-421. [CrossRef]

86. Einhorn-Stoll, U. Pectin-water interactions in foods-From powder to gel. Food Hydrocoll. 2018, 78, 109-119. [CrossRef]

87. Ravindran, R.; Jaiswal, A.K. Exploitation of Food Industry Waste for High-Value Products. Trends Biotechnol. 2016, 34, 58-69. [CrossRef]

88. Axel, C.; Zannini, E.; Arendt, E.K. Mold spoilage of bread and its biopreservation: A review of current strategies for bread shelf life extension. Crit. Rev. Food Sci. Nutr. 2017, 57, 3528-3542. [CrossRef]

89. Amoah, I.; Cairncross, C.; Sturny, A.; Rush, E. Towards improving the nutrition and health of the aged: The role of sprouted grains and encapsulation of bioactive compounds in functional bread-A review. Int. J. Food Sci. Technol. 2018, 54, 1435-1447. [CrossRef]

90. Han, W.; Ma, S.; Li, L.; Zheng, X.; Wang, X. Rheological properties of gluten and gluten-starch model doughs containing wheat bran dietary fibre. Int. J. Food Sci. Technol. 2018, 53, 2650-2656. [CrossRef] 
91. Thakur, G.; Mitra, A.; Pal, K.; Rousseau, D. Effect of flaxseed gum on reduction of blood glucose and cholesterol in type 2 diabetic patients. Int. J. Food Sci. Nutr. 2009, 60, 126-136. [CrossRef]

92. Kristensen, M.; Savorani, F.; Christensen, S.; Engelsen, S.B.; Bugel, S.; Toubro, S.; Tetens, I.; Astrup, A. Flaxseed dietary fibers suppress postprandial lipemia and appetite sensation in young men. Nutr. Metab. Cardiovasc. Dis. 2013, 23, 136-143. [CrossRef] [PubMed]

93. Altunkaya, A.; Hedegaard, R.V.; Brimer, L.; Gokmen, V.; Skibsted, L.H. Antioxidant capacity versus chemical safety of wheat bread enriched with pomegranate peel powder. Food Funct. 2013, 4, 722-727. [CrossRef] [PubMed]

94. Dall'Asta, M.; Bresciani, L.; Calani, L.; Cossu, M.; Martini, D.; Melegari, C.; Del Rio, D.; Pellegrini, N.; Brighenti, F.; Scazzina, F. In Vitro Bioaccessibility of Phenolic Acids from a Commercial Aleurone-Enriched Bread Compared to a Whole Grain Bread. Nutrients 2016, 8, 42. [CrossRef] [PubMed]

95. Koistinen, V.M.; Katina, K.; Nordlund, E.; Poutanen, K.; Hanhineva, K. Changes in the phytochemical profile of rye bran induced by enzymatic bioprocessing and sourdough fermentation. Food Res. Int. 2016, 89, 1106-1115. [CrossRef]

96. Koistinen, V.M.; Nordlund, E.; Katina, K.; Mattila, I.; Poutanen, K.; Hanhineva, K.; Aura, A.M. Effect of bioprocessing on the in vitro colonic microbial metabolism of phenolic acids from rye bran fortified breads. J. Agric. Food Chem. 2017, 65, 1854-1864. [CrossRef]

97. Sho, C.; Kurata, R.; Okuno, H.; Takase, Y.; Yoshimoto, M. Sweet potato-derived shochu distillery by-products supernatants. Nippon Shokuhin Kagaku Kogaku Kaishi 2008, 55, 287-292. [CrossRef]

98. Angelino, D.; Cossu, M.; Marti, A.; Zanoletti, M.; Chiavaroli, L.; Brighenti, F.; Del Rio, D.; Martini, D. Bioaccessibility and bioavailability of phenolic compounds in bread: A review. Food Funct. 2017, 8, 2368-2393. [CrossRef]

99. Van Dokkum, W.; Wesstra, A.; Schippers, F.A. Physiological effects of fibre-rich types of bread. Br. J. Nutr. 1982, 47, 451. [CrossRef]

100. Van Dokkum, W.; Pikaar, N.A.; Thissen, J.T.N.M. Physiological effects of fibre-rich types of bread. Br. J. Nutr. 1983, 50, 61. [CrossRef]

101. Bresciani, L.; Scazzina, F.; Leonardi, R.; Dall'Aglio, E.; Newell, M.; Dall'Asta, M.; Melegari, C.; Ray, S.; Brighenti, F.; Del Rio, D. Bioavailability and metabolism of phenolic compounds from wholegrain wheat and aleurone-rich wheat bread. Mol. Nutr. Food Res. 2016, 60, 2343-2354. [CrossRef]

102. Lu, Z.X.; Walker, K.Z.; Muir, J.G.; Mascara, T.; O’Dea, K. Arabinoxylan fiber, a byproduct of wheat flour processing, reduces the postprandial glucose response in normoglycemic subjects. Am. J. Clin. Nutr. 2000, 71, 1123-1128. [CrossRef] [PubMed]

103. Hanhineva, K.; Keski-Rahkonen, P.; Lappi, J.; Katina, K.; Pekkinen, J.; Savolainen, O.; Timonen, O.; Paananen, J.; Mykkanen, H.; Poutanen, K. The postprandial plasma rye fingerprint includes benzoxazinoid-derived phenylacetamide sulfates. J. Nutr. 2014, 144, 1016-1022. [CrossRef] [PubMed]

104. Juntunen, K.S.; Laaksonen, D.E.; Poutanen, K.S.; Niskanen, L.K.; Mykkänen, H.M. High-fiber rye bread and insulin secretion and sensitivity in healthy postmenopausal women. Am. J. Clin. Nutr. 2003, 77, 385-391. [CrossRef] [PubMed]

105. Abraham, A.; Kattoor, A.J.; Saldeen, T.; Mehta, J.L. Vitamin E and its anticancer effects. Crit. Rev. Food Sci. Nutr. 2018, 59, 1-8. [CrossRef]

106. Gao, X.; Wang, Y.; Sun, G. High dietary choline and betaine intake is associated with low insulin resistance in the Newfoundland population. Nutrition 2017, 33, 28-34. [CrossRef]

107. Wu, W.H.; Chen, T.Y.; Lu, R.W.; Chen, S.T.; Chang, C.C. Benzoxazinoids from Scoparia dulcis (sweet broomweed) with antiproliferative activity against the DU-145 human prostate cancer cell line. Phytochemistry 2012, 83, 110-115. [CrossRef]

(C) 2020 by the authors. Licensee MDPI, Basel, Switzerland. This article is an open access article distributed under the terms and conditions of the Creative Commons Attribution (CC BY) license (http://creativecommons.org/licenses/by/4.0/). 